\title{
Higgs production at the large hadron collider: Phenomenological model and theoretical predictions
}

\section{Citation}

Gastmans, R., Sau Lan Wu, and Tai Tsun Wu. 2011. "Higgs Production at the Large Hadron Collider: Phenomenological Model and Theoretical Predictions." Nuclear Physics B 850, no. 1: 53-95.

\section{Published Version}

doi:10.1016/j.nuclphysb.2011.04.012

\section{Permanent link}

http://nrs.harvard.edu/urn-3:HUL.InstRepos:12712854

\section{Terms of Use}

This article was downloaded from Harvard University's DASH repository, and is made available under the terms and conditions applicable to Open Access Policy Articles, as set forth at http:// nrs.harvard.edu/urn-3:HUL.InstRepos:dash.current.terms-of-use\#OAP

\section{Share Your Story}

The Harvard community has made this article openly available.

Please share how this access benefits you. Submit a story.

\section{Accessibility}




\title{
Higgs Production at the Large Hadron Collider: Phenomenological Model and Theoretical Predictions
}

\author{
R. Gastmans ${ }^{\mathrm{a}, 1, *}$, Sau Lan $\mathrm{Wu}^{\mathrm{b}, 2}$, Tai Tsun $\mathrm{Wu}^{\mathrm{c}, \mathrm{d}}$ \\ ${ }^{a}$ Instituut voor Theoretische Fysica, Katholieke Universiteit Leuven, Celestijnenlaan 200D, B-3001 Leuven, \\ Belgium \\ ${ }^{b}$ Department of Physics, University of Wisconsin, Madison WI 53706, USA \\ ${ }^{c}$ Gordon McKay Laboratory, Harvard University, Cambridge MA 02138, USA \\ ${ }^{d}$ Theory Division, CERN, CH-1211 Geneva 23, Switzerland
}

\begin{abstract}
Using the results from relativistic gauge field theory, a phenomenological model is developed for the production of an isolated Higgs particle $H$. The specific process is $p+p \rightarrow A+H+B$, where the group of particles $A(B)$ mostly goes down one (the other) beam pipe. The theory and the phenomenology apply when the center-of-mass energy $\sqrt{s} \gg M \gg m$, with $M$ and $m$ the masses of the Higgs particle and the proton respectively. Thus, there are two large parameters, namely $\sqrt{s} / M$ and $M / m$. That $M \gg m$ plays a central role. This phenomenological model is applied to the Large Hadron Collider (LHC) to predict the differential production cross sections. With high probability, this isolated Higgs particle is produced with a small transverse momentum of the order of $1 \mathrm{GeV} / c$. Because of this fact and the relatively small number of observed particles in such events, the method of data analysis is different from those developed so far for Higgs detection. These events can be described as due to Pomeron-Pomeron annihilation, and are 'clean' in the sense that those from TeV linear colliders are called 'clean'. The LHC, with its center-of-mass design energy of $14 \mathrm{TeV}$ and its design luminosity of $10^{34} \mathrm{~cm}^{-2} \mathrm{~s}^{-1}$, can function exceptionally well as a Pomeron collider.
\end{abstract}

Keywords: high-energy proton-proton scattering, Higgs production, LHC physics

\footnotetext{
*Corresponding author

Email address: raymond.gastmans@fys.kuleuven.be (R. Gastmans)

${ }^{1}$ Work supported in part by the FWO-Vlaanderen, project G.0651.11, and in part by the Federal Office for Scientific, Technical and Cultural Affairs through the 'Interuniversity Attraction Poles Programme Belgian Science Policy' P6/11-P.

${ }^{2}$ Work supported in part by the United States Department of Energy Grant No. DE-FG02-95ER40896.
} 


\section{Introduction}

There are two related motivations for the present study, one experimental and a second one theoretical.

The first motivation is due to the present situation in experimental high-energy physics. The Large Hadron Collider (LHC) at CERN is a proton-proton collider with a center-of-mass design energy of $14 \mathrm{TeV}$. On March 30, 2010, LHC became operational at $7 \mathrm{TeV}$, half of the design energy. This $7 \mathrm{TeV}$ is already more than three and a half times the previous maximum energy, achieved at the Fermilab Tevatron. In contrast, no electron-positron collider in the $\mathrm{TeV}$ range is expected to be built in the next decade. It is therefore natural to ask the question: to what extent is it possible to perform experiments on a high-energy protonproton machine so that they give information similar to those expected from events on an electron-positron machine?

This desire may be explained in more detail as follows. It is usually emphasized that the events from a $\mathrm{TeV} e^{+} e^{-}$accelerator are relatively 'clean', while those from a protonproton accelerator are 'messy'. The question above would be answered in the affirmative if a subclass of relatively 'clean' events can be found at LHC. When this is the situation, it is likely that it is possible to extract from this subclass of events information that is similar to that expected from an electron-positron collider.

The second motivation comes from some theoretical considerations of forty years ago. At that time, in an attempt to understand scattering processes at very high energies by studying relativistic quantum gauge field theory, it was found, among other results, that all hadron-hadron total cross sections must increase with energy [1], contrary to the general belief at that time. Three years later, this theoretical result was verified experimentally at the proton-proton collider Intersecting Storage Rings (ISR) at CERN [2]. These increasing total and integrated differential cross sections, however, have remained isolated facts since then. The question is: is there an entire class of new phenomena at high energies of which that of increasing cross sections constitutes one of the aspects?

One of the many ways to characterize elastic scattering, for example that of proton on proton for definiteness, is that these events have the largest rapidity gap [3], namely the rapidity gap between the two outgoing protons. If in addition a third particle is produced, then there are three particles in the final state. The smallest of their rapidity gaps is maximal if this third particle is produced nearly at rest in the center-of-mass system, while the two outgoing protons are in the forward and backward directions.

When these two motivations, coming from rather different physical considerations, are combined together with the present interest in the Higgs particle [4], then we are led to the Higgs production process at the Large Hadron Collider:

$$
p+p \rightarrow p+H+p .
$$

As already stated, in this process, the Higgs particle has only moderate momentum in the center-of-mass system, while the two outgoing protons are nearly in the forward and the backward directions respectively. This process satisfies the first motivation since it is an exclusive process and therefore expected to be 'clean'; it also satisfies the second motivation because of the large rapidity gaps between the protons and the Higgs particle. 
In a separate paper [5], this production process (1) has been studied from the point of view of relativistic quantum gauge field theory. This study was carried out in close analogy with the earlier treatment of elastic fermion-fermion scattering at high energies [6]: in both cases, the basic idea is to sum the leading asymptotic terms for every order of perturbation theory. For elastic scattering [6], the process is

$$
f+f \rightarrow f+f
$$

and asymptotic means

the mass of the fermion (proton)

$\ll$ center-of-mass energy of the incident fermions (protons).

For Higgs production [5], the process is

$$
f+f \rightarrow f+H+f
$$

and asymptotic means

the mass of the fermion (proton) $\ll$ mass of the Higgs particle

$\ll$ center-of-mass energy of the incident fermions (protons).

As one can expect, the case (5) with two large parameters is much more difficult to treat than the case (3) with only one large parameter.

In order to apply the field-theoretic results for the high-energy limit to future measurements at the Large Hadron Collider, it is essential to develop a phenomenological model. This is one of the main purposes of the present paper; for this, there is however a serious difficulty that must be overcome. The phenomenological model for high-energy elastic scattering used previously to predict the increase of hadronic total cross sections, such as that for the proton-proton case, has been formulated on the basis of (i) the behavior at very high energies from field theory, and (ii) the experimental data on proton-proton interactions at lower energies [6]. For the present case of the Higgs production process (1), there is no experimental data at any energy. This means that the present phenomenology must depend heavily on that for proton-proton elastic scattering. For this reason, a review of the elastic phenomenology is to be given in sec. 2 .

One more complication ought to be mentioned. At present, neither of the large detectors at the Large Hadron Collider - ATLAS and CMS - is equipped to measure protons in the nearly forward directions. This situation is expected to improve when Roman pots for the LHCf and TOTEM become operational. Without these Roman pots, it is inefficient to identify experimentally the production process (1). Since, in this process (1), the interest is more on the Higgs particle $H$ rather than on the two outgoing protons, an equally or perhaps more important process from the experimental point of view is

$$
p+p \rightarrow A+H+B
$$


where most of the group of particles $A$ go down one beam pipe, and those of group $B$ go down the other beam pipe. In this process (6), the Higgs particle $H$, or rather its decay products, constitutes the event signature.

Aside from avoiding the difficulty of observing the two outgoing protons, the process (6) has one more advantage over process (1). In order for this subclass of relatively clean events at LHC to be of importance in physics, they must satisfy some further conditions. These conditions include:

(a) The cross section for producing these events is not too small;

(b) These events have the potential of leading to new and interesting physics; and

(c) This subclass of events has qualitatively distinctive features.

For the process (1), these conditions (b) and (c) are clearly satisfied. On the other hand, it is questionable whether (a) is satisfied, because the cross section is likely to be small. A comparison of the Higgs production processes (1) and (6) shows immediately that, in addition to not having the experimental problem of detecting the two forward and backward protons, process (6) has a much larger cross section than process (1). This point is to be discussed in detail in sec. 7 .

This paper is not the first one to present a phenomenology for the 'double-Pomeron' Higgs production in $p$ - $p$ scattering through the processes (1) and (6). After the pioneering work by Schäfer, Nachtmann, and Schöpf [7], and by Müller and Schramm [8], there were several later developments as described, e.g., in ref. [9]. The present paper differs from these works in several important respects:

(a) The present method depends critically on the presence of two independent large parameters instead of one - see (2);

(b) The information from proton-proton elastic scattering plays a central role here; and

(c) In the previous work, the gluon distribution function is of central importance, but it has been completely avoided here, the reason having been given recently [10].

An earlier version of the present paper appeared as a 2009 CERN preprint [11].

\section{Phenomenology for Proton-Proton Elastic Scattering}

Shortly after the theoretical prediction of increasing cross sections was obtained from relativistic quantum field theory, experimentalists raised a most relevant and proper question: could an estimate be given for the amount of increase in the proton-proton total cross section, so that they could plan their experiment? Thus, the first phenomenological model was born, giving a rough estimate of $3 \mathrm{mb}$ for this increase from its minimum to the value at $\sqrt{s}=53 \mathrm{GeV}$ for the ISR. This crude estimate turned out to be about $2 / 3$ of the later observed increase, and it was considered a theoretical triumph.

Since then, the phenomenological model has been improved greatly $[12,13]$. Because it is the basis of the phenomenology to be presented here for the Higgs production process, 
this section is devoted to a discussion of the model for proton-proton elastic scattering. In this model, the elastic scattering amplitude is taken to be

$$
\mathcal{M}\left(s, \vec{\Delta}_{\perp}\right)=\frac{i s}{2 \pi} \int d \vec{x}_{\perp} e^{-i \vec{\Delta}_{\perp} \cdot \vec{x}_{\perp}}\left\{1-\exp \left[-\Omega\left(s, \vec{x}_{\perp}^{2}\right)\right]\right\},
$$

with

$$
\Omega\left(s, \vec{x}_{\perp}^{2}\right)=\mathcal{S}\left(\frac{s}{m^{2}}\right) F\left(\vec{x}_{\perp}^{2}\right),
$$

where $\mathcal{S}\left(s / m^{2}\right)$ is given by the crossing symmetric form

$$
\mathcal{S}\left(\frac{s}{m^{2}}\right)=\frac{\left(s / m^{2}\right)^{c}}{\left[\ln \left(s / m^{2}\right)\right]^{c^{\prime}}}+\frac{\left(u / m^{2}\right)^{c}}{\left[\ln \left(u / m^{2}\right)\right]^{c^{\prime}}},
$$

where $u$ is the third Mandelstam variable [14]. In both the 1979 and the 1984 phenomenological models [12], the Fourier transform of $F\left(x_{\perp}^{2}\right)$ is chosen to be given by

$$
\tilde{F}(t)=f[G(t)]^{2} \frac{a^{2}+t}{a^{2}-t}
$$

where $G(t)$ is essentially the electromagnetic form factor of the proton

$$
G(t)=\frac{1}{\left(1-\frac{t}{m_{1}^{2}}\right)\left(1-\frac{t}{m_{2}^{2}}\right)}
$$

There are six phenomenological parameters for proton-proton elastic scattering, namely $c$, $c^{\prime}, m_{1}, m_{2}, f$, and $a$.

Eq. (10) needs to be discussed in detail. That $\tilde{F}(t)$ should be given approximately by the square of the electromagnetic form factor was known before, and it is also known that this relation cannot be exact [15]. This is the reason why $\left(a^{2}+t\right) /\left(a^{2}-t\right)$, the extra factor in eq. (10), is considered to be acceptable. Since this parameter $a$ is about $2 \mathrm{GeV}$, this extra factor affects mostly the region where $-t \gtrsim 4 \mathrm{GeV}^{2}$; indeed, it was originally introduced to smooth out the proton-proton differential cross section in this region. There is no known physical interpretation for this factor $\left(a^{2}+t\right) /\left(a^{2}-t\right)$.

Attention is now turned to the topic of the present paper on the formulation of a phenomenological model for the Higgs production processes (1) and (6) at the Large Hadron Collider. As already pointed out in the Introduction, there is no relevant data that can be used for Higgs production, and, therefore, this phenomenology must depend on the knowledge of proton-proton elastic scattering. The formulation of a phenomenological model is not solely a matter of logical deduction; it must depend, to a large extent, on physical intuition. That the factor $\left(a^{2}+t\right) /\left(a^{2}-t\right)$ in eq. (10) has no physical interpretation makes it very difficult to incorporate into the Higgs phenomenology. Indeed, with this factor included, a great deal of effort has been spent on using the model as described by eqs. (7) to (11) for the present purpose, and all the results seem artificial. It is therefore decided to drop this 
factor $\left(a^{2}+t\right) /\left(a^{2}-t\right)$ in building the model to be presented here. See subsection 8.2 for further discussions on this point.

Accordingly, eq. (10) is replaced by

$$
\tilde{F}(t)=f[G(t)]^{2}
$$

Thus, the proton-proton elastic amplitude at high energies is taken to be given by eqs. (7), (8), (9), (11), and (12). There are now five parameters $c, c^{\prime}, m_{1}, m_{2}$, and $f$. Their values are taken without modification from the 1984 paper [12], namely

$$
\begin{aligned}
c & =0.167, \\
c^{\prime} & =0.748 \\
m_{1} & =0.586 \mathrm{GeV}, \\
m_{2} & =1.704 \mathrm{GeV}, \\
f & =7.115 \mathrm{GeV}^{-2} .
\end{aligned}
$$

Actually, in many of the papers on hadron-hadron elastic scattering at high energies, Regge backgrounds [16] due to the exchange of various particles are included; the parameters listed here pertain only to the Pomeron [17]. For the present study on Higgs production at the Large Hadron Collider, again due to the absence of experimental data at any energy, it does not seem possible to include such Regge backgrounds. This is one of the many related problems that remains to be solved in the future.

\section{Formulation of the Phenomenological Model - Step 1 (Preliminaries)}

Because of the absence of any experimental data for the process (1) or (6), any phenomenological model for Higgs production at the Large Hadron Collider must rely heavily on the results of calculations from relativistic gauge field theory. These results have been presented in ref. [5]. Although the fermion in that treatment lacks internal structure, it is nevertheless identified with the proton for phenomenological purposes.

Let $m$ and $M$ denote respectively the masses of the proton and the Higgs particle. From the electron-positron colliding accelerator LEP at CERN, there is preliminary experimental evidence that $M$ is about $115 \mathrm{GeV} / c^{2}[18,19]$. Thus, the condition (5) can be written as

$$
m \ll M \ll \sqrt{s}
$$

and is expected to be well satisfied at the Large Hadron Collider. The Higgs production process (1) is shown schematically in fig. 1, where the four-momenta of the incoming protons are denoted by $p_{1}$ and $p_{2}$, while those in the final state by $p_{1}^{\prime}$ and $p_{2}^{\prime}$. Thus, there are two momentum transfers

$$
\Delta_{1}=p_{1}-p_{1}^{\prime}, \quad \text { and } \quad \Delta_{2}=p_{2}-p_{2}^{\prime},
$$

and the momentum of the produced Higgs particle is

$$
p_{H}=\Delta_{1}+\Delta_{2} .
$$




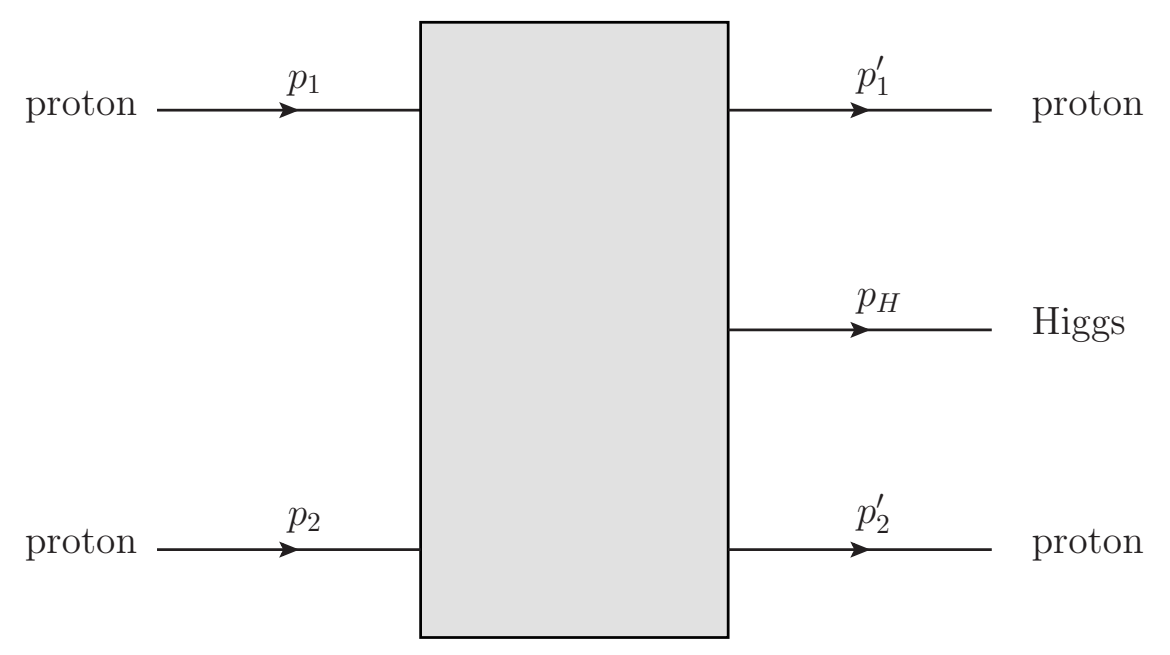

Figure 1: Schematic representation of the Higgs production process $p+p \rightarrow p+H+p$.

At the Large Hadron Collider, the two incoming protons interact head-on; it is convenient to take the spatial direction of one of these protons as the $z$-axis. With this notation, the specific kinematic region of interest is

$$
\vec{\Delta}_{1 \perp}=\mathcal{O}(m), \quad \vec{\Delta}_{2 \perp}=\mathcal{O}(m)
$$

and

$$
p_{H z}=\mathcal{O}(M) \text {. }
$$

This means that all transverse momenta are of the order of $1 \mathrm{GeV} / c$, and the velocity of the produced Higgs particle in the center-of-mass system is moderate.

Because of (18), it is convenient to apply a Lorentz transformation in the $z$-direction to go to a new frame where

$$
p_{H z}=0 .
$$

We shall work in this new frame, where the energies of the two incoming protons are no longer equal. Let these energies be called $\omega_{1}$ and $\omega_{2}$, then

$$
s \sim 4 \omega_{1} \omega_{2}
$$

and both $\omega_{1}$ and $\omega_{2}$ are of the order of $\sqrt{s}$. Of course, under such a Lorentz transformation, the perpendicular components are not changed and, hence, (17) remains valid.

In developing the phenomenology for Higgs production in this and the following sections, it is often essential to indicate which result holds for field theory and which one for phenomenology. In this and the next three sections, the following notation is to be used:

(i) If a relation holds for field theory, then the equation number is followed by " $\mathrm{f}$ "; if it holds for phenomenology, then the letter "p" is used.

(ii) An arrow indicates that the transition from a relation in field theory to phenomenology, i.e., the left-hand side of an arrow $\rightarrow$ is for field theory, while the right-hand side is for phenomenology. 
It may be useful to illustrate this notation in the context of proton-proton elastic scattering. In this much simpler case, the leading term of order $s(\ln s)^{n}$ comes from a well-defined set of diagrams with $n$ four-fermion loops, the tower diagrams, and the summation over $n$ of these leading terms gives

$$
\mathcal{M}_{T} \sim i s\left\langle J\left|\left(\frac{s}{m^{2}}\right)^{\mathcal{K}_{t}}\right| J\right\rangle
$$

where

$$
J\left(\vec{q}_{\perp}, \vec{\Delta}\right)=\frac{g^{2}}{2 m}\left(\vec{q}_{\perp}^{2}+\lambda^{2}\right)^{-1 / 2}\left[\left(\vec{\Delta}-\vec{q}_{\perp}\right)^{2}+\lambda^{2}\right]^{-1 / 2}
$$

and $\mathcal{K}_{t}$ is a fairly complicated operator, of which the details are not of concern here. The relevant important property of this $\mathcal{K}_{t}$ is that, for physical values of the momentum transfer $\vec{\Delta}$, it is a self-adjoint operator with a continuum spectrum whose upper limit is a positive number independent of $\vec{\Delta}$. The quantity $g$ in eq. (22f) is the coupling constant of the quantum field theory used to derive (21f) and the two-dimensional vector $\vec{q}_{\perp}$ is the integration variable.

How can we develop a phenomenology for proton-proton elastic scattering on the basis of (21f) and (22f) from relativistic quantum gauge field theory? First of all, since eq. (22f) from field theory does not capture the structure of the proton in terms of three quarks, it cannot be used at all for the phenomenology. We are therefore left with (21f) only. Using the properties of the operator $\mathcal{K}_{t}$ given in the last paragraph, the transition to phenomenology is accomplished by

$$
\left\langle J\left|\left(\frac{s}{m^{2}}\right)^{\mathcal{K}_{t}}\right| J\right\rangle \rightarrow S\left(\frac{s}{m^{2}}\right) \tilde{F}\left(-\vec{\Delta}_{\perp}^{2}\right)
$$

where

$$
S\left(\frac{s}{m^{2}}\right)=\frac{\left(s / m^{2}\right)^{c}}{\left[\ln \left(s / m^{2}\right)\right]^{c^{\prime}}},
$$

because of the spectrum structure of the operator $\mathcal{K}_{t}$. Actually, this form (24) holds from summing the leading terms of the perturbation series in field theory, except here the values of $c$ and $c^{\prime}$ are taken to be different from those of field theory.

In the phenomenology, crossing symmetry must be taken into account, and therefore eq. (24) must be replaced by (9), i.e.,

$$
\mathcal{S}\left(\frac{s}{m^{2}}\right)=\frac{\left(s / m^{2}\right)^{c}}{\left[\ln \left(s / m^{2}\right)\right]^{\prime}}+\frac{\left(u / m^{2}\right)^{c}}{\left[\ln \left(u / m^{2}\right)\right]^{c^{\prime}}},
$$

and hence (23) is replaced by

$$
\left\langle J\left|\left(\frac{s}{m^{2}}\right)^{\mathcal{K}_{t}}\right| J\right\rangle \rightarrow \mathcal{S}\left(\frac{s}{m^{2}}\right) \tilde{F}\left(-\vec{\Delta}_{\perp}^{2}\right) .
$$

Of course, the quantities $\tilde{F}\left(-\vec{\Delta}_{\perp}^{2}\right)$ in $(23)$ and (26) are not quite the same. Finally, the expressions (10) [or (12)] and (11) are entirely for phenomenology, and have nothing to do with the tower diagrams.

In summary, for proton-proton elastic scattering and a number of similar cases, the transition from relativistic quantum gauge field theory to phenomenology is accomplished 


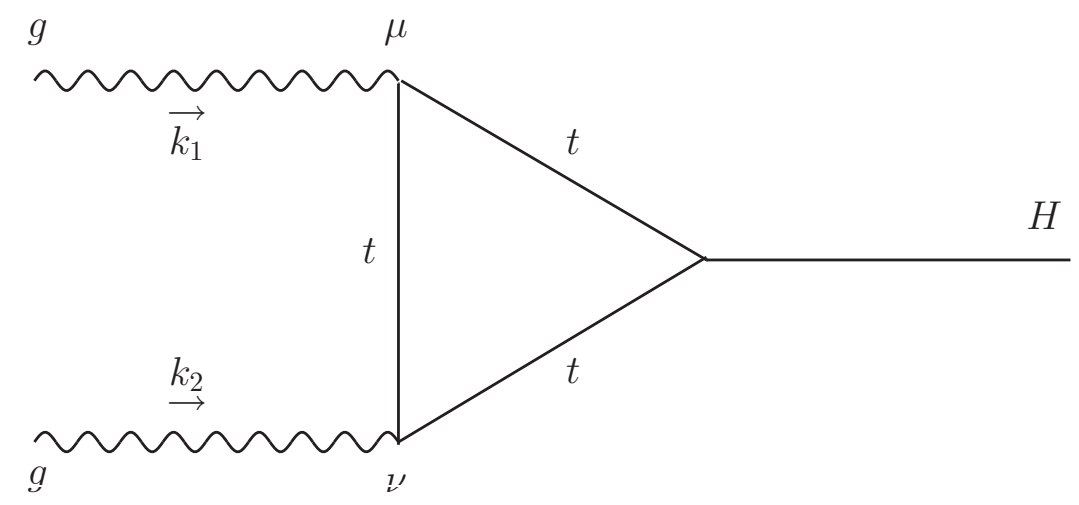

Figure 2: The top triangular diagram for Higgs production by gluon fusion.

entirely through (26). All the other necessary information comes from experimental data at lower energies.

Attention is now turned to the Higgs production process (1) at the Large Hadron Collider. As already emphasized, for this process and unlike the cases of elastic scattering, there is no experimental data for this (1) at any energy. For this reason alone, the development of a phenomenology for Higgs production at the Large Hadron Collider is necessarily much more complicated. This development will be carried out step by step in the next four sections.

\section{Formulation of a Phenomenological Model — Step 2}

In order to formulate a phenomenological model for the Higgs production process (1) $p+p \rightarrow p+H+p$ at high energies, the first question to be answered is: What is the relevant information that can be used as inputs to this model? The absence of experimental data on this process means that it is necessary to rely extensively on results from relativistic quantum gauge theory. By summing the leading terms of the perturbation series for this production process, it has been found [5] that its matrix element is approximately given by

$$
I \sim-s\left\langle\left(\frac{2 \omega_{2}}{M}\right)^{\mathcal{K}_{t}} J|V|\left(\frac{2 \omega_{1}}{M}\right)^{\mathcal{K}_{t}} J\right\rangle
$$

where $J$ is given by eq. (22f) and, from the massless nature of the gluon,

$$
V=C_{0} \frac{\left(\vec{q}_{0 \perp}+\vec{\Delta}_{1 \perp}\right) \cdot\left(\vec{q}_{0 \perp}-\vec{\Delta}_{2 \perp}\right)}{\left\|\vec{q}_{0 \perp}+\vec{\Delta}_{1 \perp}\right\|\left\|\vec{q}_{0 \perp}-\vec{\Delta}_{2 \perp}\right\|} .
$$

In this eq. (28f), the two-dimensional vector $\vec{q}_{0 \perp}$ is the integration variable and the constant $C_{0}$ comes from the top triangle of fig. 2 and is given by [20]

$$
C_{0}=\frac{i g_{s}^{2} g_{e w}}{(2 \pi)^{2}} \frac{m_{t}^{2}}{m_{W}} \int_{0}^{1} d \gamma_{1} \int_{0}^{1-\gamma_{1}} d \gamma_{2} \frac{1-4 \gamma_{1} \gamma_{2}}{m_{t}^{2}-\gamma_{1} \gamma_{2} M^{2}-i \epsilon}
$$


where $g_{s}$ and $g_{e w}$ are the usual strong and electroweak coupling constants, while $m_{t}$ and $m_{W}$ are the masses of the top quark and the $W$ boson respectively. An explicit expression for $C_{0}$ in terms of elementary functions can be found in ref. [21].

Actually, (27f) is somewhat symbolic, but suffices for the present purpose. The two quantities $J$ are not quite the same: the one on the left depends on the momentum transfer $\vec{\Delta}_{2 \perp}$ to the fermion (proton) with incident energy $\omega_{2}$, while $J$ on the right depends on the corresponding $\vec{\Delta}_{1 \perp}$ to the other proton. Similarly, the two $\mathcal{K}_{t}$ in this (27f) are not the same, the $\mathcal{K}_{t}$ on the left (right) depends on $\vec{\Delta}_{2 \perp}\left(\vec{\Delta}_{1 \perp}\right)$. That these two $\mathcal{K}_{t}$ are different in general has profound consequences - see sec. 6 .

As already stated in sec. 3, eq. (22f) from field theory cannot be used for phenomenology. Under this circumstance, the only source of information that can be used to incorporate the proton structure into (27f) is from proton-proton scattering described in sec. 2 . More specifically, there is no choice but to supplement (27f) by the replacement (26), where $\mathcal{S}\left(\mathrm{s} / \mathrm{m}^{2}\right)$ is defined by eq. (9) and involves the two parameters $c$ and $c^{\prime}$, while $\tilde{F}(t)$ with $t=-\vec{\Delta}_{\perp}^{2}$ is given by eqs. (11) and (12), involving the parameters $m_{1}, m_{2}$, and $f$. The values of these five parameters are given by eqs. (13).

In as much as the proton-proton-Pomeron vertex $J$ of eq. (22f) cannot be used in phenomenology, the question is immediately raised whether the Pomeron-Pomeron-Higgs vertex $V$ of eq. (28f) can be used in phenomenology.

Clearly, the two cases of the $J$ and the $V$ are very different. As already stated in sec. 3, eq. (22f) for $J$ fails to capture the structure of the proton as three quarks. On the other hand, the $V$ as given by eqs. (28f) and (29) does describe accurately the diagram of fig. 2 for Higgs production by gluon fusion. This shows that the difficulty with the $J$ of eq. (22f) does not occur with the $V$ of eqs. (28f) and (29). It is true that there are radiative corrections to this diagram of fig. 2 , but such corrections are not expected to modify eq. (28f) significantly. First, strong interactions are included in the operator $\mathcal{K}_{t}$, at least partially. Secondly, the factor $C_{0}$ of eq. (29), up to radiative corrections that are not included, describes the gluon fusion process

$$
g+g \rightarrow H
$$

and has been used to estimate Higgs production. Note that strong corrections inside the top triangle are presumably small, unlike the corrections to $J$. Of course, it would be most interesting if one could explicitly calculate and incorporate the corrections beyond leading order as is done for various semi-hard processes in general. On the basis of these arguments, eqs. (28f) and (29) for the Pomeron-Pomeron-Higgs vertex are going to be used not only in field theory but also in the phenomenology.

In summary, the following information is to be used as inputs in developing the phenomenology for the Higgs production process (1).

(i) The field-theoretic result (27f) is used for the matrix element of (1), $p+p \rightarrow p+H+p$. This (27f) is similar in nature to (21f) for proton-proton elastic scattering; neither (21f) nor (27f) can be used directly in the phenomenology.

(ii) The replacement (26) is used to take the proton-proton-Pomeron vertex correctly into account in the phenomenology. 
(iii) Eqs. (28f) and (29) are accepted as sufficiently accurate for the phenomenology. Actually, this (iii) is quite complicated and will be discussed further in secs. 6 and 7 .

This list of inputs already shows a serious difficulty in formulating a phenomenology for the Higgs production process (1) in terms of the known parameters for proton-proton elastic scattering.

As discussed in sec. 3, the replacement (26) - see (ii) above - is the totality of all the information that can be obtained from the knowledge on proton-proton elastic scattering. In other words, since it is only possible to make a transition from field theory to phenomenology for the diagonal element $\left\langle J, s^{\mathcal{K}_{t}} J\right\rangle$, how can we get any handle on the right-hand side of (27f)? This problem is especially serious because the proton-proton-Pomeron vertex $J$ is expected to be quite complicated in view of the quark structure of the proton. For some time, efforts were made to try to guess an approximation to this $J$, but it was soon evident that making such a guess was unlikely to be successful. In other words, there seems to be no reliable way of gaining, from the phenomenology of proton-proton elastic scattering, sufficient information for either $J$ or $\left(s / M^{2}\right)^{\mathcal{K}_{t}}|J\rangle$.

\section{Formulation of a Phenomenological Model — Step 3}

Let (27f) be written out more explicitly as

$$
\begin{aligned}
& I\left(\omega_{1}, \omega_{2}, \vec{\Delta}_{1 \perp}, \vec{\Delta}_{2 \perp}\right) \\
& \quad \sim-s\left\langle\left(\frac{2 \omega_{2}}{M}\right)^{\mathcal{K}_{t}\left(\left\|\vec{\Delta}_{2 \perp}\right\|\right)} J\left(\left\|\vec{\Delta}_{2 \perp}\right\|\right)\left|V\left(\vec{\Delta}_{1 \perp}, \vec{\Delta}_{2 \perp}\right)\right|\left(\frac{2 \omega_{1}}{M}\right)^{\mathcal{K}_{t}\left(\left\|\vec{\Delta}_{1 \perp}\right\|\right)} J\left(\left\|\vec{\Delta}_{1 \perp}\right\|\right)\right\rangle,
\end{aligned}
$$

where $V\left(\vec{\Delta}_{1 \perp}, \vec{\Delta}_{2 \perp}\right)$ is given by eq. (28f). Although the serious difficulty discussed at the end of sec. 4 is present in general, there is fortunately a special case where this difficulty does not appear. The present sec. 5 is devoted to this special case.

The special case is the one where the Higgs is produced, through the process (1), with no transverse momentum, i.e.,

$$
\vec{p}_{H \perp}=0
$$

or, by eq. (16),

$$
\vec{\Delta}_{1 \perp}+\vec{\Delta}_{2 \perp}=0 \text {. }
$$

When eq. (33) is satisfied, the quantity $V$ of eq. (28f) reduces simply to

$$
V=C_{0}
$$

which is the constant given by eq. (29). Note that, in view of the discussion in sec. 4 , eq. (28f), including the present special case eq. (34), is valid not only for field theory but also approximately for phenomenology.

By eq. (34), (31f) reduces to

$$
\begin{aligned}
& -\left.s^{-1} I\left(\omega_{1}, \omega_{2}, \vec{\Delta}_{1 \perp}, \vec{\Delta}_{2 \perp}\right)\right|_{\vec{\Delta}_{1 \perp}+\vec{\Delta}_{2 \perp}=0} \\
& \left.\quad \sim C_{0}\left\langle J\left(\left\|\vec{\Delta}_{2 \perp}\right\|\right)\left(\frac{2 \omega_{2}}{M}\right)^{\mathcal{K}_{t}\left(\left\|\vec{\Delta}_{2 \perp}\right\|\right)}\left(\frac{2 \omega_{1}}{M}\right)^{\mathcal{K}_{t}\left(\left\|\vec{\Delta}_{1 \perp}\right\|\right)} J\left(\left\|\vec{\Delta}_{1 \perp}\right\|\right)\right\rangle\right|_{\vec{\Delta}_{1 \perp}+\vec{\Delta}_{2 \perp}=0}
\end{aligned}
$$


in this special case. Furthermore, since eq. (33) implies $\left\|\vec{\Delta}_{1 \perp}\right\|=\left\|\vec{\Delta}_{2 \perp}\right\|$, the two $\mathcal{K}_{t}$ 's in (35f) are the same and hence

$$
\begin{array}{rl}
-s^{-1} & \left.I\left(\omega_{1}, \omega_{2}, \vec{\Delta}_{1 \perp}, \vec{\Delta}_{2 \perp}\right)\right|_{\vec{\Delta}_{1 \perp}+\vec{\Delta}_{2 \perp}=0} \\
& \sim C_{0}\left\langle J\left(\left\|\vec{\Delta}_{1 \perp}\right\|\right)\left(\frac{2 \omega_{2}}{M}\right)^{\mathcal{K}_{t}\left(\left\|\vec{\Delta}_{1 \perp}\right\|\right)}\left(\frac{2 \omega_{1}}{M}\right)^{\mathcal{K}_{t}\left(\left\|\vec{\Delta}_{1 \perp}\right\|\right)} J\left(\left\|\vec{\Delta}_{1 \perp}\right\|\right)\right\rangle \\
& =C_{0}\left\langle J\left(\left\|\vec{\Delta}_{1 \perp}\right\|\right)\left(\frac{4 \omega_{1} \omega_{2}}{M^{2}}\right)^{\mathcal{K}_{t}\left(\left\|\vec{\Delta}_{1 \perp}\right\|\right)} J\left(\left\|\vec{\Delta}_{1 \perp}\right\|\right)\right\rangle \\
& =C_{0}\left\langle J\left(\left\|\vec{\Delta}_{1 \perp}\right\|\right)\left(\frac{s}{M^{2}}\right)^{\mathcal{K}_{t}\left(\left\|\vec{\Delta}_{1 \perp}\right\|\right)} J\left(\left\|\vec{\Delta}_{1 \perp}\right\|\right)\right\rangle .
\end{array}
$$

This is precisely the form for $\mathcal{M}_{T}$ as given by eq. (21f). The important difference is that the Higgs mass $M$ appears in the right-hand side of (36f) instead of the proton mass $m$ in (21f) for proton-proton elastic scattering. In this way, a first relation has been found between the Higgs production process (1) and the proton-proton elastic scattering:

$$
\left.I\left(\omega_{1}, \omega_{2}, \vec{\Delta}_{1 \perp}, \vec{\Delta}_{2 \perp}\right)\right|_{\vec{\Delta}_{1 \perp}+\vec{\Delta}_{2 \perp}=0} \sim i C_{0} \mathcal{M}_{T}\left(\frac{s}{M^{2}}, \vec{\Delta}_{1 \perp}\right),
$$

where $s$ is related to $\omega_{1}$ and $\omega_{2}$ by (20).

This relation (37f) from field theory is taken over also for the phenomenology without change:

$$
\left.I\left(\omega_{1}, \omega_{2}, \vec{\Delta}_{1 \perp}, \vec{\Delta}_{2 \perp}\right)\right|_{\vec{\Delta}_{1 \perp}+\vec{\Delta}_{2 \perp}=0}=i C_{0} \mathcal{M}_{T}\left(\frac{s}{M^{2}}, \vec{\Delta}_{1 \perp}\right) .
$$

Note that, while (37f) is an approximate relation from field theory, eq. (38p) is an exact relation which defines the matrix element for the Higgs production process (1) in the present phenomenology in the special case where $\vec{\Delta}_{1 \perp}+\vec{\Delta}_{2 \perp}=0$. By (26), eq. (38p) gives more explicitly

$$
\left.I\left(\omega_{1}, \omega_{2}, \vec{\Delta}_{1 \perp}, \vec{\Delta}_{2 \perp}\right)\right|_{\vec{\Delta}_{1 \perp}+\vec{\Delta}_{2 \perp}=0}=i C_{0} \mathcal{S}\left(\frac{s}{M^{2}}\right) \tilde{F}\left(-\vec{\Delta}_{1 \perp}^{2}\right),
$$

in the special case of eq. (32).

The importance of this special case cannot be over-emphasized: without this special case that leads to the relations (37f) and hence (38p) between Higgs production and elastic scattering, it is doubtful that a phenomenology can be developed for the process (1). Actually, the significance of the special case goes even further. For the process (1) and for any fixed value of the Higgs longitudinal momentum $p_{H z}$, the Higgs distribution as a function of $\vec{p}_{H \perp}^{2}$ peaks at $\vec{p}_{H \perp}=0$. If this peak value is given accurately, the percentage error of the total Higgs production through (1) does not depend critically on the accuracy off peak. In other words, when

$$
\vec{\Delta}_{1 \perp}+\vec{\Delta}_{2 \perp} \neq 0
$$

less accurate approximations are acceptable. This is to be discussed in the next section. 


\section{Formulation of the Phenomenological Model — Step 4}

In the preceding section, a relation has been found between the matrix elements for $p+p \rightarrow p+p$ and $p+p \rightarrow p+H+p$. This relation holds when $m \ll M \ll \sqrt{s}$ and $\vec{\Delta}_{1 \perp}+\vec{\Delta}_{2 \perp}=0$. The former condition is expected to be well satisfied at the Large Hadron Collider. However, to have a phenomenological model for Higgs production, it is necessary to remove the latter condition of $\vec{\Delta}_{1 \perp}+\vec{\Delta}_{2 \perp}=0$. This is to be accomplished in this section.

With reference to eq. (16), this step of going from $\vec{p}_{H \perp}=0$ to $\vec{p}_{H \perp} \neq 0$ is quite different in nature than the treatment of the case $\vec{p}_{H \perp}=0$ in the preceding section. In sec. 4 , all the available information is listed as (i), (ii), and (iii) for developing a phenomenological model. This information is, however, not sufficient to pin down the matrix element for Higgs production in general, but with a very important and useful exceptional case $\vec{\Delta}_{1 \perp}+\vec{\Delta}_{2 \perp}=0$, as discussed in the preceding sec. 5 .

Independent of the approach to the case $\vec{\Delta}_{1 \perp}+\vec{\Delta}_{2 \perp} \neq 0$, where there is insufficient information, one criterion that must be satisfied is that no discontinuity is allowed, especially at $\vec{\Delta}_{1 \perp}+\vec{\Delta}_{2 \perp}=0$, i.e.,

$$
\lim _{\vec{\Delta}_{1 \perp}+\vec{\Delta}_{2 \perp} \rightarrow 0}\left[\text { approximation for } \vec{\Delta}_{1 \perp}+\vec{\Delta}_{2 \perp} \neq 0\right]=\left[\text { approximation for } \vec{\Delta}_{1 \perp}+\vec{\Delta}_{2 \perp}=0\right],
$$

where the right-hand side is given by eq. (39p).

Two fairly drastic approximations will be introduced in this section to deal with the case $\vec{\Delta}_{1 \perp}+\vec{\Delta}_{2 \perp} \neq 0$. The first one concerns the quantity $V$, given by eq. (28f). When $\vec{\Delta}_{1 \perp}+$ $\vec{\Delta}_{2 \perp}=0$, this eq. (28f) reduces simply to (34), meaning that in this case $V$ not only does not depend on the value of $\vec{\Delta}_{1 \perp}$, but also is independent of the variable $\vec{q}_{0 \perp}$. Indeed, it is this property that makes the development of sec. 5 possible. When $\vec{\Delta}_{1 \perp}+\vec{\Delta}_{2 \perp} \neq 0$, the right-hand side of eq. (28f), on the contrary, is a function of $\vec{\Delta}_{1 \perp}, \vec{\Delta}_{2 \perp}$, and $\vec{q}_{0 \perp}$. The first fairly dramatic approximation is that eq. (34) is to be used even when $\vec{\Delta}_{1 \perp}+\vec{\Delta}_{2 \perp} \neq 0$, i.e.,

$$
V\left(\vec{q}_{0 \perp}, \vec{\Delta}_{1 \perp}, \vec{\Delta}_{2 \perp}\right) \sim C_{0}
$$

A comparison with the exact equality (28f) shows that this approximation (42p) does satisfy the criterion (41p).

When this approximation (42p) is used, eq. (31f) becomes

$$
\begin{aligned}
& I\left(\omega_{1}, \omega_{2}, \vec{\Delta}_{1 \perp}, \vec{\Delta}_{2 \perp}\right) \\
& \quad \sim-C_{0} s\left\langle J\left(\| \vec{\Delta}_{2 \perp}||\right)\left|\left(\frac{2 \omega_{2}}{M}\right)^{\mathcal{K}_{t}\left(\left\|\vec{\Delta}_{2 \perp}\right\|\right)}\left(\frac{2 \omega_{1}}{M}\right)^{\mathcal{K}_{t}\left(\left\|\vec{\Delta}_{1 \perp}\right\|\right)}\right| J\left(\left\|\vec{\Delta}_{1 \perp}\right\|\right)\right\rangle .
\end{aligned}
$$

When $\vec{\Delta}_{1 \perp}+\vec{\Delta}_{2 \perp}=0$, further simplifications occur as shown in sec. 5 , because the two $\mathcal{K}_{t}$ 's on the right-hand side are the same. In order to approximate the right-hand side of eq. (43f), a second approximation is used. 
The basic idea of this second approximation is to make use of inequalities. The Schwarz inequality is one of the best known inequalities, i.e.,

$$
\left|\int_{a}^{b} d x f(x) g(x)\right|^{2} \leq\left[\int_{a}^{b} d x|f(x)|^{2}\right]\left[\int_{a}^{b} d x|g(x)|^{2}\right]
$$

where $b>a$. For the present purpose, let $f(x)$ and $g(x)$ both be positive. Then,

$$
\left[\int_{a}^{b} d x f(x) g(x)\right]^{2} \leq\left[\int_{a}^{b} d x f(x)^{2}\right]\left[\int_{a}^{b} d x g(x)^{2}\right]
$$

If $f(x)$ and $g(x)$ are sufficiently similar, then this inequality (45) can be used as an approximation

$$
\int_{a}^{b} d x f(x) g(x) \sim \sqrt{\left[\int_{a}^{b} d x f(x)^{2}\right]\left[\int_{a}^{b} d x g(x)^{2}\right]} .
$$

It should also be emphasized that, while the inequality (45) only makes sense when $f(x)$ and $g(x)$ are real, the approximation (46) may be a good one even when $f(x)$ and $g(x)$ are complex.

Suppose this approximation (46) is to be applied to the right-hand side of eq. (43f). Then, the result is

$$
\begin{aligned}
& \left\langle J\left(\| \vec{\Delta}_{2 \perp}||\right)\left|\left(\frac{2 \omega_{2}}{M}\right)^{\mathcal{K}_{t}\left(\left\|\vec{\Delta}_{2 \perp}\right\|\right)}\left(\frac{2 \omega_{1}}{M}\right)^{\mathcal{K}_{t}\left(\left\|\vec{\Delta}_{1 \perp}\right\|\right)}\right| J\left(\left\|\vec{\Delta}_{1 \perp}\right\|\right)\right\rangle
\end{aligned}
$$

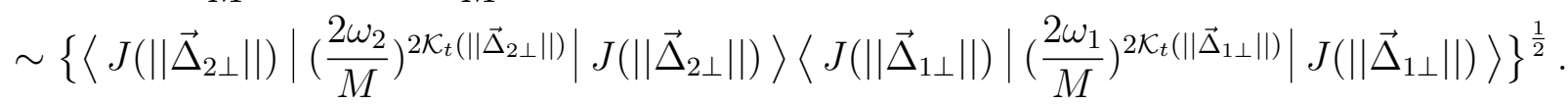

However, this approximation is not acceptable. The reason is that, in the limit $\vec{\Delta}_{1 \perp}+\vec{\Delta}_{2 \perp} \rightarrow$ 0 , the right-hand side of (47f) is

$$
\left\{\left\langle J\left(\left\|\vec{\Delta}_{1 \perp}\right\|\right)\left|\left(\frac{4 \omega_{2}^{2}}{M^{2}}\right)^{\mathcal{K}_{t}\left(\left\|\vec{\Delta}_{1 \perp}\right\|\right)}\right| J\left(\left\|\vec{\Delta}_{1 \perp}\right\|\right)\right\rangle\left\langle J\left(\left\|\vec{\Delta}_{1 \perp}\right\|\right)\left|\left(\frac{4 \omega_{1}^{2}}{M^{2}}\right)^{\mathcal{K}_{t}\left(\left\|\vec{\Delta}_{1 \perp}\right\|\right)}\right| J\left(\| \vec{\Delta}_{1 \perp}||\right)\right\rangle\right\}^{\frac{1}{2}},
$$

which is in general not equal to

$$
\left\langle J\left(\| \vec{\Delta}_{1 \perp}||\right)\left|\left(\frac{4 \omega_{1} \omega_{2}}{M^{2}}\right)^{\mathcal{K}_{t}\left(\left\|\vec{\Delta}_{1 \perp}\right\|\right)}\right| J\left(\left\|\vec{\Delta}_{1 \perp}\right\|\right)\right\rangle .
$$

This violates the criterion (41p). This shows the power of eq. (41p).

In order to satisfy condition (41p), a generalized form of the Schwarz inequality, or Hölder's inequality, should be used. One way to write this inequality (not in its most general form) is

$$
\int_{a}^{b} d x f(x)^{\alpha} g(x)^{\beta} \leq\left[\int_{a}^{b} d x f(x)^{\alpha+\beta}\right]^{\frac{\alpha}{\alpha+\beta}}\left[\int_{a}^{b} d x g(x)^{\alpha+\beta}\right]^{\frac{\beta}{\alpha+\beta}}
$$


where again $b>a$, and $f(x), g(x), \alpha$, and $\beta$ are all positive. This inequality (48) reduces to (45) when $\alpha=\beta=1$. Similar to the case (46), this (48) is turned into an approximation in the form

$$
\int_{a}^{b} d x f(x)^{\alpha} g(x)^{\beta} \sim\left[\int_{a}^{b} d x f(x)^{\alpha+\beta}\right]^{\frac{\alpha}{\alpha+\beta}}\left[\int_{a}^{b} d x g(x)^{\alpha+\beta}\right]^{\frac{\beta}{\alpha+\beta}}
$$

In order to use this approximation on the right-hand side of eq. (43f), let

$$
\begin{aligned}
f(x) & =e^{\mathcal{K}_{t}\left(\left\|\vec{\Delta}_{2 \perp}\right\|\right)}, \\
g(x) & =e^{\mathcal{K}_{t}\left(\left\|\vec{\Delta}_{1 \perp}\right\|\right)}, \\
\alpha & =\ln \frac{2 \omega_{2}}{M}, \\
\text { and } \quad \beta & =\ln \frac{2 \omega_{1}}{M} .
\end{aligned}
$$

It then follows from eq. (49) that

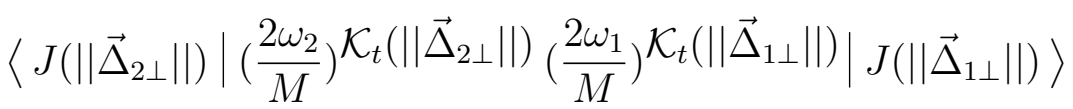

$$
\begin{aligned}
& \sim\left\langle J\left(\left\|\vec{\Delta}_{2 \perp}\right\|\right)\left|e^{\mathcal{K}_{t}\left(\| \vec{\Delta}_{2 \perp}||\right)\left(\ln \frac{2 \omega_{2}}{M}+\ln \frac{2 \omega_{1}}{M}\right)}\right| J\left(\| \vec{\Delta}_{2 \perp}||\right)\right\rangle^{\frac{\ln \frac{2 \omega_{2}}{M}}{\ln \frac{2 \omega_{2}}{M}+\ln \frac{2 \omega_{1}}{M}}} \\
& \times\left\langle J\left(\| \vec{\Delta}_{1 \perp}||\right)\left|e^{\mathcal{K}_{t}\left(\left\|\vec{\Delta}_{1 \perp}\right\|\right)\left(\ln \frac{2 \omega_{1}}{M}+\ln \frac{2 \omega_{2}}{M}\right)}\right| J\left(\left\|\vec{\Delta}_{1 \perp}\right\|\right)\right\rangle^{\frac{\ln \frac{2 \omega_{1}}{M}}{\ln \frac{2 \omega_{1}}{M}+\ln \frac{2 \omega_{2}}{M}}},
\end{aligned}
$$

or

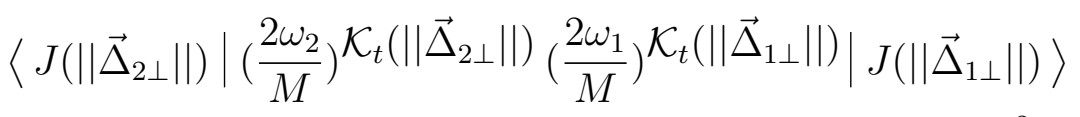

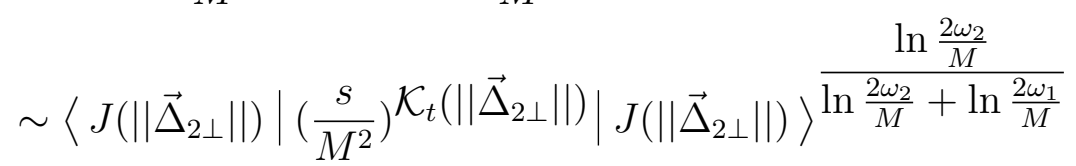

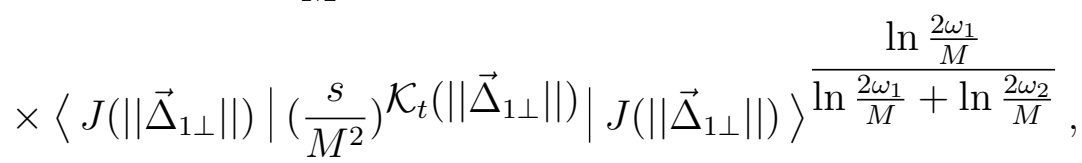

where eq. (20) has been used. Finally, with eq. (21f), the substitution of (55f) into eq. (43f) yields

$$
\begin{aligned}
& I\left(\omega_{1}, \omega_{2}, \vec{\Delta}_{1 \perp}, \vec{\Delta}_{2 \perp}\right) \\
& \quad \sim-C_{0}\left[-i \mathcal{M}_{T}\left(\frac{s}{M^{2}}, \vec{\Delta}_{1 \perp}\right)\right]^{\frac{\ln \frac{2 \omega_{1}}{M}}{\ln \frac{2 \omega_{1}}{M}+\ln \frac{2 \omega_{2}}{M}}}\left[-i \mathcal{M}_{T}\left(\frac{s}{M^{2}}, \vec{\Delta}_{2 \perp}\right)\right]^{\frac{\ln \frac{2 \omega_{2}}{M}}{\ln \frac{2 \omega_{2}}{M}+\ln \frac{2 \omega_{2}}{M}}} .
\end{aligned}
$$


For general $\vec{\Delta}_{1 \perp}$ and $\vec{\Delta}_{2 \perp}$, this is the desired expression. It is the best that can be done under the present circumstance of not having any experimental data for the production process (1) at any energy.

The meaning of (56) is similar to that of (37f) and (38p): it is a rough approximation in field theory, but is taken to be accurate in the phenomenological model so that it defines the left-hand side quantity $I\left(\omega_{1}, \omega_{2}, \vec{\Delta}_{1 \perp}, \vec{\Delta}_{2 \perp}\right)$.

This approximate expression (56) has the following peculiarity; however, this peculiarity has been judged to be acceptable. While the function $I$ on the left-hand side depends on $\vec{\Delta}_{1 \perp}$ and $\vec{\Delta}_{2 \perp}$, i.e., on $\left\|\vec{\Delta}_{1 \perp}\right\|,\left\|\vec{\Delta}_{2 \perp}\right\|$, and $\left(\vec{\Delta}_{1 \perp} \cdot \vec{\Delta}_{2 \perp}\right)$, the right-hand side does not have any dependence on $\left(\vec{\Delta}_{1 \perp} \cdot \vec{\Delta}_{2 \perp}\right)$. This lack of dependence has the consequence that the approximate (56) reduces to (37f) and (38p) not only when $\vec{\Delta}_{1 \perp}+\vec{\Delta}_{2 \perp}=0$, but, more generally, when $\left\|\vec{\Delta}_{1 \perp}\right\|=\left\|\vec{\Delta}_{2 \perp}\right\|$. It is due to the first approximation as expressed by (42p), of which the right-hand side is just a constant, while the left-hand side given by eq. (28f) depends on $\left(\vec{\Delta}_{1 \perp} \cdot \vec{\Delta}_{2 \perp}\right)$.

\section{Phenomenological Model}

On the basis of the fairly accurate approximation of sec. 5 and the less accurate approximation of sec. 6 , an approximation (56) has been obtained which expresses $I\left(\omega_{1}, \omega_{2}, \vec{\Delta}_{1 \perp}, \vec{\Delta}_{2 \perp}\right)$ in terms of the elastic scattering amplitude $\mathcal{M}_{T}$. With this relation and the discussion in sec. 2 on proton-proton elastic scattering, we are in a position to write down a phenomenological model for the Higgs production process (1) as follows.

In terms of the energy $\omega$ of the incoming protons in the center-of-mass system, define

$$
\omega_{1}=\frac{\omega}{M}\left(\sqrt{M^{2}+p_{H z}^{2}}-p_{H z}\right)
$$

and

$$
\omega_{2}=\frac{\omega}{M}\left(\sqrt{M^{2}+p_{H z}^{2}}+p_{H z}\right) .
$$

These eqs. (57) are consequences of the Lorentz transformation used to achieve (19). In this sec. 7 , since most of the formulas refer to the phenomenological model, the notation "p" as defined in sec. 3 is omitted.

The combination of the approximate relation (56) derived in sec. 6 with eqs. (9) and (12) from proton-proton elastic scattering leads to the definition of the quantity

$$
\mathcal{A}=C_{0} \mathcal{S}\left(\frac{s}{M^{2}}\right) f\left[G\left(-\vec{\Delta}_{1 \perp}^{2}\right)\right]^{\frac{2 \ln \frac{2 \omega_{1}}{M}}{\ln \frac{2 \omega_{1}}{M}+\ln \frac{2 \omega_{2}}{M}}}\left[G\left(-\vec{\Delta}_{2 \perp}^{2}\right)\right]^{\frac{2 \ln \frac{2 \omega_{2}}{M}}{\ln \frac{2 \omega_{1}}{M}+\ln \frac{2 \omega_{2}}{M}}}
$$

where

$$
\mathcal{S}(y)=\frac{y^{c}}{(\ln y)^{c^{\prime}}}+\frac{y^{c} e^{-i \pi c}}{(\ln y-i \pi)^{c^{\prime}}}
$$




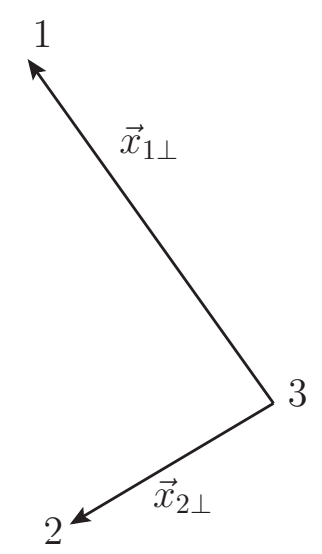

Figure 3: Impact distances in the transverse coordinate system. The points 1 and 2 denote the positions of the two incoming protons, while the point 3 is for that of the produced Higgs particle. Only relative distances are physically meaningful.

is essentially the same as the function $\mathcal{S}$ defined by eq. (9). Following again eq. (7) from proton-proton elastic scattering, define $\overline{\mathcal{A}}$ to be the double Fourier transform of this quantity $\mathcal{A}$, or, explicitly,

$$
\overline{\mathcal{A}}\left(\vec{x}_{1 \perp}, \vec{x}_{2 \perp}\right)=\frac{1}{2 \pi} \int d \vec{\Delta}_{1 \perp} e^{i \vec{\Delta}_{1 \perp} \cdot \vec{x}_{1 \perp}} \frac{1}{2 \pi} \int d \vec{\Delta}_{2 \perp} e^{i \vec{\Delta}_{2 \perp} \cdot \vec{x}_{2 \perp}} \mathcal{A}\left(\vec{\Delta}_{1 \perp}, \vec{\Delta}_{2 \perp}\right) .
$$

These impact distances $\vec{x}_{1 \perp}$ and $\vec{x}_{2 \perp}$ in the transverse plane are shown in fig. 3 . Again following eq. (7), this function $\overline{\mathcal{A}}\left(\vec{x}_{1 \perp}, \vec{x}_{2 \perp}\right)$ must be modified by two attenuation factors $\Omega$, leading to

$$
\tilde{\mathcal{A}}\left(\vec{x}_{1 \perp}, \vec{x}_{2 \perp}\right)=e^{-\mathcal{S}\left(\frac{2 \omega_{1}}{M}\right) F\left(\vec{x}_{1 \perp}^{2}\right)} e^{-\mathcal{S}\left(\frac{2 \omega_{2}}{M}\right) F\left(\vec{x}_{2 \perp}^{2}\right)} \overline{\mathcal{A}}\left(\vec{x}_{1 \perp}, \vec{x}_{2 \perp}\right) .
$$

This is the analog of the quantity $1-\exp \left[-\Omega\left(s, x_{\perp}^{2}\right)\right]$ of eq. (7). The matrix element for the Higgs production process (1) is then given by

$$
M E\left(p_{H z}, \vec{\Delta}_{1 \perp}, \vec{\Delta}_{2 \perp}\right)=\frac{1}{2 \pi} \int d \vec{x}_{1 \perp} e^{-i \vec{\Delta}_{1 \perp} \cdot \vec{x}_{1 \perp}} \frac{1}{2 \pi} \int d \vec{x}_{2 \perp} e^{-i \vec{\Delta}_{2 \perp} \cdot \vec{x}_{2 \perp}} \tilde{\mathcal{A}}\left(\vec{x}_{1 \perp}, \vec{x}_{2 \perp}\right) .
$$

This is finally the analog of eq. (7) from proton-proton elastic scattering. From this matrix element (62), the various cross sections are essentially given by its squared absolute value as usual.

Actually, the cross section obtained in this way is not the cross section for the process (1). To get the cross section for this process (1), an additional factor of

$$
e^{-\mathcal{S}\left(\frac{s}{m^{2}}\right) F\left(\left(\vec{x}_{1 \perp}-\vec{x}_{2 \perp}\right)^{2}\right)}
$$

must be included in the $\tilde{\mathcal{A}}\left(\vec{x}_{1 \perp}, \vec{x}_{2 \perp}\right)$ of eq. (61), again in complete analogy with eq. (7). From the point of view of physics, it is, however, the expression (61) for $\tilde{\mathcal{A}}\left(\vec{x}_{1 \perp}, \vec{x}_{2 \perp}\right)$, without this 


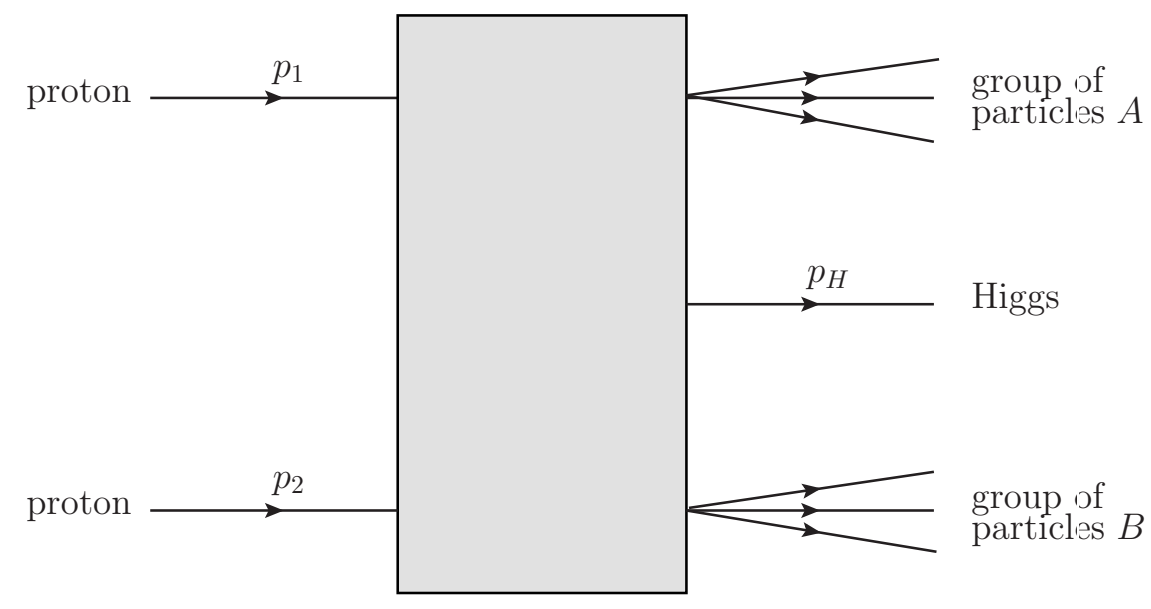

Figure 4: Schematic representation of the Higgs production process $p+p \rightarrow A+H+B$.

factor (63), that should be used in eq. (62). The reason is as follows. After the Higgs particle is produced through the process (1), there is only a small probability for the protons to remain protons, and precisely this small probability is described by the factor (63). For the experiments at the Large Hadron Collider, there are two reasons why this factor (63) should not be included. First, since it is the purpose to study the properties of the Higgs particle, it does not matter whether the two protons remain protons or not. Secondly, even if we are interested in the process where the two protons remain protons, none of the detectors at the Large Hadron Collider is equipped to detect efficiently, at least at present, the protons in the very forward or backward directions, as was discussed in the Introduction.

In short, the cross section obtained by the present phenomenological model is indeed the physically interesting cross section. It is not for the process (1); it is instead the cross section for the Higgs production process

$$
p+p \rightarrow A+H+B
$$

where $A$ consists of one or more particles produced by one of the incoming protons, while $B$ is a similar group of particles produced by the other incoming proton. Instead of fig. 1, the process (64) is shown schematically in fig. 4. From the experimental point of view, the group of particles $A$ mostly go down one beam pipe and, similarly, the group of particles $B$ mostly go down the other beam pipe. Occasionally, some particles from either $A$ or $B$ may get out of the respective beam pipes, but these particles are expected to have relatively small transverse momenta and can thus be identified.

It only remains to simplify somewhat expression (62) for the matrix element relevant to the production process (64). As seen from (62), the matrix element $M E\left(p_{H z}, \vec{\Delta}_{1 \perp}, \vec{\Delta}_{2 \perp}\right)$ is in the form of an eightfold integral. Because of rotational symmetry, this eightfold integral can be reduced to a fourfold integral involving Bessel functions $J_{0}$. Furthermore, the quantity $\mathcal{A}$ of eq. (58) is the product of two functions, one depending on $\vec{\Delta}_{1 \perp}$ and the other on $\vec{\Delta}_{2 \perp}$. Thus, the fourfold integral for $M E\left(p_{H z}, \vec{\Delta}_{1 \perp}, \vec{\Delta}_{2 \perp}\right)$ is actually the product of two double 
integrals. When this reduction is carried out, the result is

$$
\begin{aligned}
& M E\left(p_{H z}, \vec{\Delta}_{1 \perp}, \vec{\Delta}_{2 \perp}\right)=C_{0} \mathcal{S}\left(\frac{s}{M^{2}}\right) f \\
& \times\left\{\int_{0}^{\infty} d x_{1} x_{1} J_{0}\left(x_{1}|| \vec{\Delta}_{1 \perp}||\right) e^{-\mathcal{S}\left(\frac{2 \omega_{1}}{M}\right) F\left(x_{1}^{2}\right)} \int_{0}^{\infty} d \xi_{1} \xi_{1} J_{0}\left(x_{1} \xi_{1}\right)\left[G\left(-\xi_{1}^{2}\right)\right]^{\left.\frac{2 \ln \frac{2 \omega_{1}}{M}}{\ln \frac{2 \omega_{1}}{M}+\ln \frac{2 \omega_{2}}{M}}\right\}}\right. \\
& \times\left\{\int_{0}^{\infty} d x_{2} x_{2} J_{0}\left(x_{2}|| \vec{\Delta}_{2 \perp}||\right) e^{-\mathcal{S}\left(\frac{2 \omega_{2}}{M}\right) F\left(x_{2}^{2}\right)} \int_{0}^{\infty} d \xi_{2} \xi_{2} J_{0}\left(x_{2} \xi_{2}\right)\left[G\left(-\xi_{2}^{2}\right)\right]^{\left.\frac{2 \ln \frac{2 \omega_{2}}{M}}{\ln \frac{2 \omega_{1}}{M}+\ln \frac{2 \omega_{2}}{M}}\right\} .}\right.
\end{aligned}
$$

This is the formula to be used to give theoretical predictions for the Higgs production process (64) at the Large Hadron Collider.

In this formula (65), the function $\mathcal{S}$ is defined by eq. (59), the function $F\left(x^{2}\right)$ is given by the inverse Fourier transform of $\tilde{F}(t)$ of eq. (12), the function $J_{0}$ is the Bessel function of order 0 , the function $G$ is given by eq. (11), and $\omega_{1}$ and $\omega_{2}$ by eqs. (57a) and (57b), while the coefficient $C_{0}$ of eq. (29) comes from the top triangle of gluon fusion as shown in fig. 2 . The parameters are taken from proton-proton elastic scattering and given in eqs. (13), while $M$ is the Higgs mass.

\section{Phenomenological Model - Discussions}

The formulation of the phenomenological model described in the preceding sec. 7 has been a complicated process. It is the purpose of the present sec. 8 to discuss some of the choices made, the rationale for these choices, and their consequences on the limitations on the phenomenological model.

\subsection{Insufficient inputs}

As already discussed in the Introduction, the major difficulty of formulating a phenomenological model for the Higgs production process (1) is that there is no experimental data on this process at any energy. The difficulty is seen more clearly in sec. 4 from the list (i), (ii), and (iii) of available information as inputs to the model.

It has turned out that this lack of information is not fatal. This is because of the special case where the Higgs particle is produced with vanishing transverse momentum. In this special case where $\vec{\Delta}_{1 \perp}+\vec{\Delta}_{2 \perp}=0-$ see eqs. (32) and (33) - the matrix element for Higgs production at high energies is given approximately by a corresponding one for proton-proton elastic scattering. This fortunate special case is studied in sec. 5 .

It is even more fortunate that this special case corresponds to the peak of the transverse Higgs distribution. Once this peak cross section is known, less accurate off-peak values are acceptable. This matrix element for Higgs production at the peak is given in sec. 5, and that for off-peak in sec. 6. In this way, a phenomenological model is obtained in sec. 7. 


\subsection{Parameter a from elastic scattering}

Because of the absence of experimental data for Higgs production in proton-proton collisions at any energy, the present phenomenology depends heavily on that for proton-proton elastic scattering. In sec. 2, where this elastic phenomenology is described, there are six independent parameters [12]. One of the six parameters, $a$, is neglected for the Higgs phenomenology of sec. 7 . What are the reasons for neglecting this parameter?

There are several good reasons for neglecting this $a$, the first two having been mentioned in sec. 2 .

(a) There is no known physical interpretation for this parameter.

(b) This factor $\left(a^{2}+t\right) /\left(a^{2}-t\right)$ in eq. (10) affects mostly the region where the momentum transfer $\left\|\vec{\Delta}_{\perp}\right\|$ is of the order of $2 \mathrm{GeV} / c$ or larger. At such momentum transfers, the elastic differential cross section $d \sigma / d t$, for example at the ISR energies, has gone down about six orders of magnitude compared with that in the forward direction (neglecting Coulomb corrections). In contrast, the Higgs phenomenology, developed here and summarized in sec. 7, cannot be expected to be accurate for the Higgs transverse momentum of the order of $2 \mathrm{GeV} / c$.

(c) Besides the physical reasons of (a) and (b), there is in addition a technical reason for omitting this factor $\left(a^{2}+t\right) /\left(a^{2}-t\right)$. For the special case of $\vec{\Delta}_{1 \perp}+\vec{\Delta}_{2 \perp}=0$ treated in sec. 5 , there is no difficulty in keeping this factor. However, since

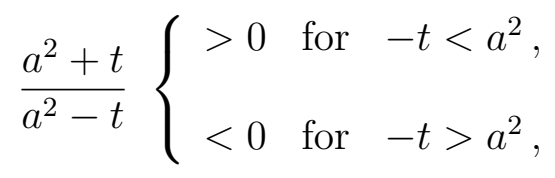

the method developed in sec. 6 for the more general case of $\vec{\Delta}_{1 \perp}+\vec{\Delta}_{2 \perp} \neq 0$ no longer works if this factor $\left(a^{2}+t\right) /\left(a^{2}-t\right)$ is kept, the reason being the failure of Hölder's inequality when the integrand is not non-negative. Efforts to avoid using Hölder's inequality have not been successful.

\subsection{Approximate formula for $V$}

The next issue to be discussed is the approximation (42p) for the Pomeron-Pomeron-Higgs vertex. This approximation bears some resemblance to the omission of the parameter $a$, as discussed in subsection 8.2, but there are also major differences.

By (34), this (42p) is actually exact when $\vec{\Delta}_{1 \perp}+\vec{\Delta}_{2 \perp}=0$. Thus, (42p) does not enter in the treatment of sec. 5; rather, it is used in sec. 6 to go from the special case $\vec{\Delta}_{1 \perp}+$ $\vec{\Delta}_{2 \perp}=0$ to the general case where $\vec{\Delta}_{1 \perp}$ and $\vec{\Delta}_{2 \perp}$ are independent. Because of the lack of experimental data, this extension in sec. 6 is based on the use of Hölder's inequality (48) as an approximation expressed by (49). When the $f(x)$ and $g(x)$ of (48) are sufficiently similar, this approximation (49) is expected to be accurate.

In the application of (49) to the matrix element for Higgs production, a good approximation may be expected when $\vec{\Delta}_{1 \perp}+\vec{\Delta}_{2 \perp}$ is relatively small. For this reason, by eq. (16), the present phenomenology is accurate not only at the peak point $\vec{p}_{H \perp}=0$, as already discussed 
in the last paragraph of sec. 5 , but also for small values of $\vec{p}_{H \perp}$. This means that the peak region of the Higgs distribution is well described by this phenomenology. As this distribution decreases rapidly as a function of $\vec{p}_{H \perp}^{2}$, the lack of accuracy for larger values of $\vec{p}_{H \perp}^{2}$ has little effect on the Higgs production cross section integrated over $\vec{p}_{H \perp}$. This is also the reason why the approximation (42p) can be used.

On the other hand, the use of (49) cannot be expected to yield an accurate approximation when $\vec{\Delta}_{1 \perp}$ and $-\vec{\Delta}_{2 \perp}$ are vastly different. Since $\vec{\Delta}_{1 \perp}$ and $\vec{\Delta}_{2 \perp}$ are independent variables, there are important regions where they are very different. This point will be studied further in the next subsection 8.4.

In summary, we are of the opinion that it is well justified to omit the parameter $a$ as discussed in subsection 8.2, i.e., the replacement of the factor $\left(a^{2}+t\right) /\left(a^{2}-t\right)$ by 1 so that eq. (10) simplifies to eq. (12). On the other hand, it seems possible that the approximation (42p) will be improved in the future.

\subsection{Range of validity for the phenomenological model}

In the phenomenological model described in sec. 7 , there are two prominent exponents see eq. (58) or (65); for the purpose of the discussion here, let the notations be introduced:

$$
\alpha_{1}=2 \frac{\ln \frac{2 \omega_{1}}{M}}{\ln \frac{2 \omega_{1}}{M}+\ln \frac{2 \omega_{2}}{M}}
$$

and

Clearly,

$$
\alpha_{2}=2 \frac{\ln \frac{2 \omega_{2}}{M}}{\ln \frac{2 \omega_{1}}{M}+\ln \frac{2 \omega_{2}}{M}} .
$$

$$
\alpha_{1}+\alpha_{2}=2 .
$$

As seen from eq. (58), $\alpha_{1}\left(\alpha_{2}\right)$ is the exponent of a quantity that depends on $\vec{\Delta}_{1 \perp}^{2}\left(\vec{\Delta}_{2 \perp}^{2}\right)$. When $\vec{\Delta}_{1 \perp}+\vec{\Delta}_{2 \perp}=0$, or, more generally, when $\left\|\vec{\Delta}_{1 \perp}\right\|=\left\|\vec{\Delta}_{2 \perp}\right\|$, the right-hand side of eq. (58) depends only on the sum $\alpha_{1}+\alpha_{2}$, which is 2 by eq. (69), meaning that the values of $\alpha_{1}$ and $\alpha_{2}$ separately do not matter. When $\left\|\vec{\Delta}_{1 \perp}\right\|$ and $\left\|\vec{\Delta}_{2 \perp}\right\|$ are not equal, then the quantity $\mathcal{A}$ of eq. (58) depends on what $\alpha_{1}$ and $\alpha_{2}$ are. Therefore, an important question is: for this phenomenology model, what is the range of validity for $\alpha_{1}$ and $\alpha_{2}$ ? This question has been touched upon in the preceding subsection 8.3.

Since eq. (58) comes from (56), which is obtained by applying Hölder's inequality to the special case $\left\|\vec{\Delta}_{1 \perp}\right\|=\left\|\vec{\Delta}_{2 \perp}\right\|$, the most dangerous region for the validity of (56) is where $\left\|\vec{\Delta}_{1 \perp}\right\|$ and $\left\|\vec{\Delta}_{2 \perp}\right\|$ are very different from each other. For definiteness, let $\left\|\vec{\Delta}_{1 \perp}\right\|$ be much larger than $\left\|\vec{\Delta}_{2 \perp}\right\|$. In this case, consider the factor

$$
\left[G\left(-\vec{\Delta}_{1 \perp}^{2}\right)\right]^{\alpha_{1}}=\left[\left(1+\frac{\vec{\Delta}_{1 \perp}^{2}}{m_{1}^{2}}\right)\left(1+\frac{\vec{\Delta}_{1 \perp}^{2}}{m_{2}^{2}}\right)\right]^{-\alpha_{1}}
$$

by eq. (11). Because of eq. (65), this quantity appears in the integral

$$
\int_{0}^{\infty} d \xi_{1} \xi_{1} J_{0}\left(x_{1} \xi_{1}\right)\left[\left(1+\frac{\xi_{1}^{2}}{m_{1}^{2}}\right)\left(1+\frac{\xi_{1}^{2}}{m_{2}^{2}}\right)\right]^{-\alpha_{1}}
$$


In the most symmetric case, $\alpha_{1}$ and $\alpha_{2}$ are both equal to 1 because of eq. (69). When $\alpha_{1}=1$, this integral (71) is convergent for all non-negative values of $x_{1}$. This remains true when $\alpha_{1}$ is larger than 1 .

The situation is qualitatively different when $\alpha_{1}$ is less than 1 : for $\alpha_{1}>1 / 2$, this integral (71) remains convergent as before; but for $\alpha_{1}=1 / 2$, this (71) becomes divergent at $x_{1}=0$. Since this is a qualitative change of behavior of the integral, we conclude that the phenomenological model is expected to be accurate only for

$$
\alpha_{1}>\frac{1}{2}
$$

Similarly, there is the condition

$$
\alpha_{2}>\frac{1}{2}
$$

It then follows from (67), (68), (69), (72), and (73) that

$$
\frac{1}{3}<\frac{\ln \left(2 \omega_{1} / M\right)}{\ln \left(2 \omega_{2} / M\right)}<3 .
$$

Eqs. (54) can then be used to express $\omega_{1}$ and $\omega_{2}$ in terms of $p_{H z}$, the longitudinal momentum of the produced Higgs particle. The inequalities (72) and (73) then yield

$$
\sqrt{M^{2}+p_{H z}^{2}}-\left|p_{H z}\right|>\sqrt{\frac{M^{3}}{2 \omega}},
$$

which is equivalent to

$$
2\left|p_{H z}\right|<\sqrt{2 \omega M}\left(1-\frac{M}{2 \omega}\right) .
$$

By (14), the second term on the right is negligible; therefore, this (76) reduces to

$$
\left|p_{H z}\right|<\sqrt{\frac{\omega M}{2}}
$$

This is a necessary condition for the validity of the phenomenological model: the longitudinal momentum of the produced Higgs particle in the center-of-mass system cannot be too large.

\section{Numerical Results}

Given the phenomenological amplitude (65) for Higgs production, it is now possible to make theoretical predictions for the process (64) at the Large Hadron Collider. The basic formula relating the matrix element $M E\left(p_{H z}, \vec{\Delta}_{1 \perp}, \vec{\Delta}_{2 \perp}\right)$ to the physical distributions is

$$
\frac{d^{5} \sigma}{d \vec{\Delta}_{1 \perp} d \vec{\Delta}_{2 \perp} d p_{H z}}=\frac{\left|M E\left(p_{H z}, \vec{\Delta}_{1 \perp}, \vec{\Delta}_{2 \perp}\right)\right|^{2}}{16 \pi^{3} \sqrt{M^{2}+p_{H z}^{2}}} .
$$


Since the matrix element $M E\left(p_{H z}, \vec{\Delta}_{1 \perp}, \vec{\Delta}_{2 \perp}\right)$ depends only on the lengths of the vectors $\vec{\Delta}_{1 \perp}$ and $\vec{\Delta}_{2 \perp}$, it is convenient to write

$$
M E\left(p_{H z}, \vec{\Delta}_{1 \perp}, \vec{\Delta}_{2 \perp}\right)=M E\left(p_{H z}, \Delta_{1 \perp}, \Delta_{2 \perp}\right),
$$

where

$$
\Delta_{1 \perp}=\left\|\vec{\Delta}_{1 \perp}\right\| \quad \text { and } \quad \Delta_{2 \perp}=\left\|\vec{\Delta}_{2 \perp}\right\| .
$$

Introducing the rapidity $\eta$ of the Higgs particle,

$$
\eta=\frac{1}{2} \ln \left(\frac{\sqrt{M^{2}+p_{H z}^{2}}+p_{H z}}{\sqrt{M^{2}+p_{H z}^{2}}-p_{H z}}\right),
$$

the formula (78) can be simplified to read

$$
\frac{d^{3} \sigma}{d \Delta_{1 \perp} d \Delta_{2 \perp} d \eta}=\frac{\Delta_{1 \perp} \Delta_{2 \perp}}{4 \pi}\left|M E\left(p_{H z}, \Delta_{1 \perp}, \Delta_{2 \perp}\right)\right|^{2} .
$$

A first application of the result (82) consists in obtaining the rapidity distribution $d \sigma / d \eta$. To this end, the right-hand-side of eq. (82) has to be integrated over $\Delta_{1 \perp}$ and $\Delta_{2 \perp}$, which is straightforward because of the factorized form of $M E\left(p_{H z}, \Delta_{1 \perp}, \Delta_{2 \perp}\right)$ and the simple dependence on $\Delta_{1 \perp}$ and $\Delta_{2 \perp}$. The result is

$$
\frac{d \sigma}{d \eta}=\frac{1}{16 \pi^{3}}\left|C_{0} \mathcal{S}\left(\frac{s}{M^{2}}\right) f\right|^{2} F_{1}\left(s_{1}, \alpha_{1}\right) F_{1}\left(s_{2}, \alpha_{2}\right)
$$

where the quantities $s_{1}$ and $s_{2}$ are given by [see eqs. (54)]

$$
s_{i}=2 M \omega_{i}, \quad i=1,2,
$$

the exponents $\alpha_{1}$ and $\alpha_{2}$ by eqs. (67) and (68), and the function $F_{1}$ by

$$
\begin{gathered}
F_{1}\left(s_{i}, \alpha_{i}\right)=2 \pi \int_{0}^{\infty} d x x \exp \left[-2 F\left(x^{2}\right) \operatorname{Re} \mathcal{S}\left(\frac{s_{i}}{M^{2}}\right)\right] \\
\times\left(\int_{0}^{\infty} d \xi \xi J_{0}(x \xi)\left[G\left(-\xi^{2}\right)\right]^{\alpha_{i}}\right)^{2}, i=1,2 .
\end{gathered}
$$

For an efficient numerical evaluation of the integrals, the infinite $\xi$-integral in eq. (85) is rewritten as a finite integral [22]:

$$
\begin{aligned}
\int_{0}^{\infty} d \xi & \xi J_{0}(x \xi)\left[G\left(-\xi^{2}\right)\right]^{\alpha}=\frac{\left(m_{1} m_{2}\right)^{2 \alpha}}{[\Gamma(\alpha)]^{2}}\left(\frac{x}{2}\right)^{2 \alpha-1} \\
& \times \int_{0}^{1} d s[s(1-s)]^{\alpha-1}\left[s m_{2}^{2}+(1-s) m_{1}^{2}\right]^{\frac{1-2 \alpha}{2}} K_{2 \alpha-1}\left(x \sqrt{s m_{2}^{2}+(1-s) m_{1}^{2}}\right)
\end{aligned}
$$




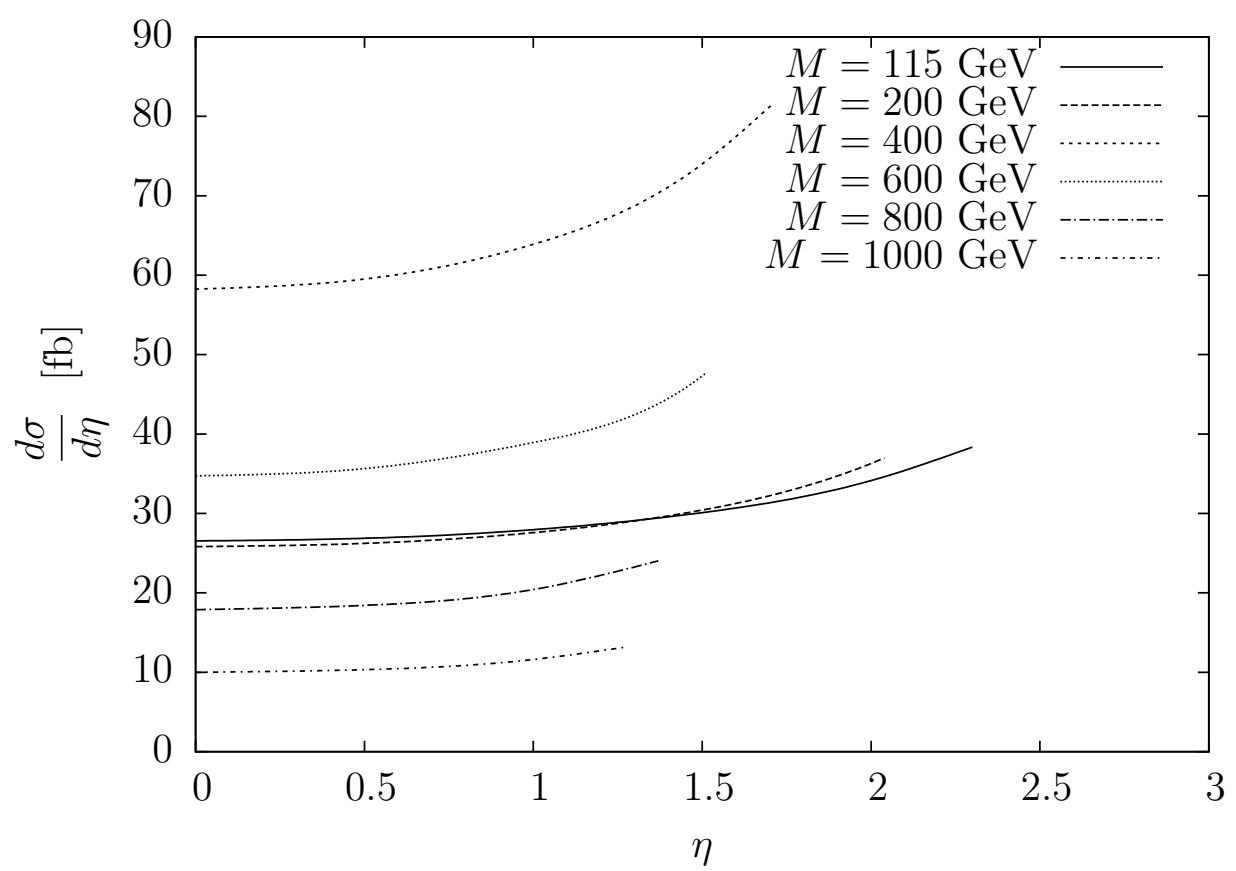

Figure 5: Rapidity distribution of the Higgs particle for various values of the Higgs mass $M$.

where $K_{\nu}$ is the modified Bessel function of the second kind. To obtain this result, use is made of the explicit formula (11) for $G(t)$. Also, the function $F\left(x^{2}\right)$ defined in eq. (12) can be expressed in terms of these Bessel functions, i.e.,

$$
\begin{aligned}
& F\left(x^{2}\right)=\frac{f\left(m_{1} m_{2}\right)^{3}}{2\left(m_{2}^{2}-m_{1}^{2}\right)^{3}} \\
& \quad \times\left\{-4 m_{1} m_{2}\left[K_{0}\left(m_{1} x\right)-K_{0}\left(m_{2} x\right)\right]+\left(m_{2}^{2}-m_{1}^{2}\right)\left[m_{2} x K_{1}\left(m_{1} x\right)+m_{1} x K_{1}\left(m_{2} x\right)\right]\right\} .
\end{aligned}
$$

The results for the rapidity distribution $d \sigma / d \eta$ are presented in figs. 5 for the Higgs mass ranging from $115 \mathrm{GeV} / c^{2}$ to $1000 \mathrm{GeV} / c^{2}$. A few comments concerning the rapidity distributions are in order:

1. The distribution $d \sigma / d \eta$ is an even function of $\eta$. Therefore, the results are only given for the positive values of $\eta$.

2. Because of the kinematical restrictions (72) and (73), the distributions are limited by $\alpha_{1}>0.52$. For larger values of the Higgs mass $M$, this endpoint is reached at smaller values of the rapidity $\eta$.

3. The distribution $d \sigma / d \eta$ does not vary much with $\eta$.

4. The experimentally accessible total cross section can be obtained approximately by integrating the rapidity distribution over the range $|\eta|<2.5$. This point will be discussed in more detail in subsection 12.2.

5. The various curves in figs. 5 first increase and then decrease with increasing $M$. This is related to the behavior of the top triangular diagram of fig. 2 [eq. (29)]. In particular, 


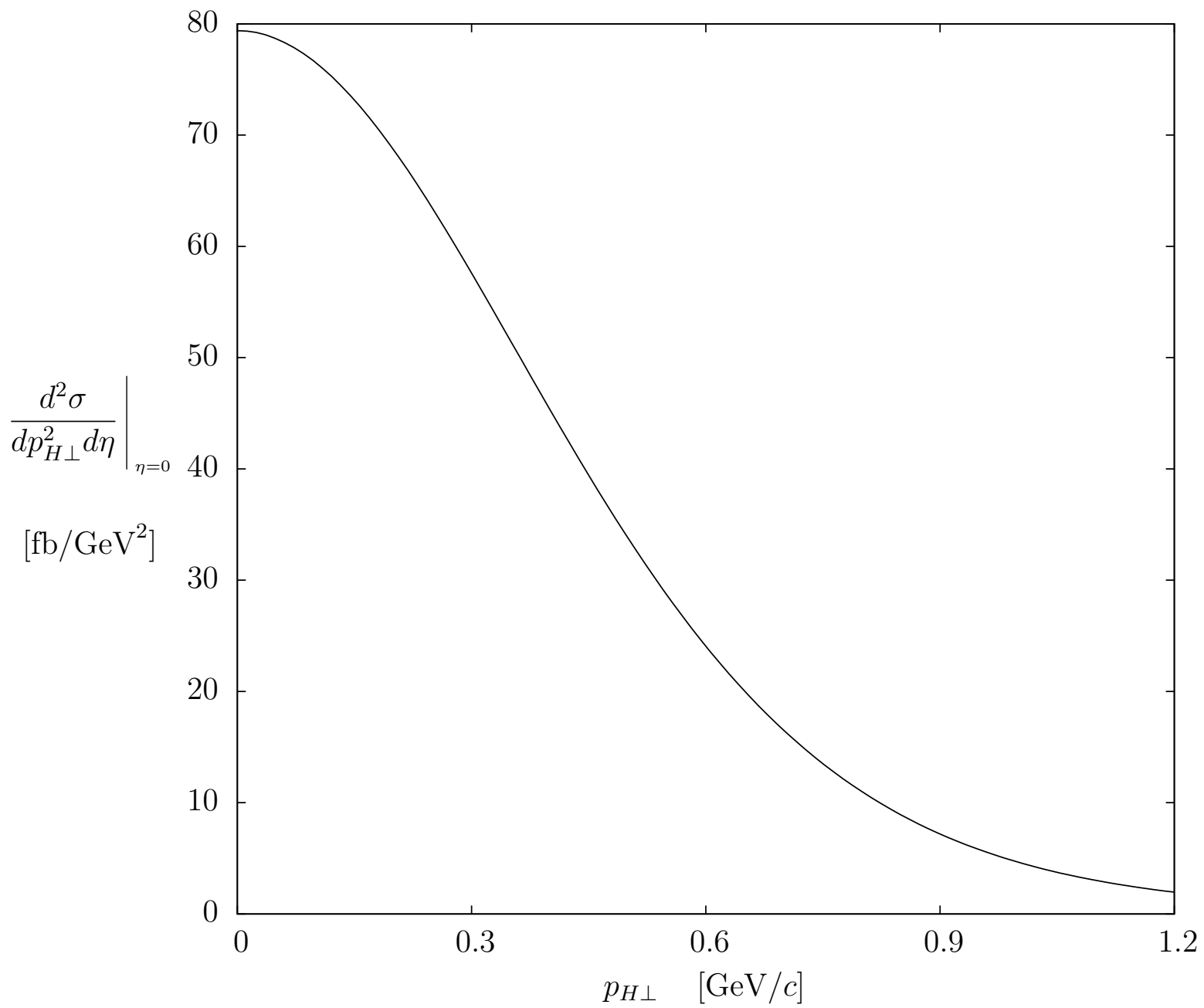

Figure 6: Distribution of the transverse momentum $p_{H \perp}$ of the Higgs particle with rapidity $\eta=0$ for the Higgs mass $M=115 \mathrm{GeV} / c^{2}$.

the highest curve corresponds to a Higgs mass of $400 \mathrm{GeV} / c^{2}$, not far away from twice the mass of the top quark.

It is also of interest to study the transverse momentum distribution of the Higgs particle. The calculation of this quantity is particularly simple in the case of zero rapidity, $\eta=0$. For that configuration, the formula reads

$$
\left.\frac{d^{2} \sigma}{d p_{H \perp}^{2} d \eta}\right|_{\eta=0}=\frac{1}{16 \pi^{3}} \int d \vec{\Delta}_{1 \perp} d \vec{\Delta}_{2 \perp} \delta^{(2)}\left(\vec{\Delta}_{1 \perp}+\vec{\Delta}_{2 \perp}-\vec{p}_{H \perp}\right)\left|M E\left(0, \vec{\Delta}_{1 \perp}, \vec{\Delta}_{2 \perp}\right)\right|^{2},
$$

where the transverse momentum vector $\vec{p}_{H \perp}$ has a length $p_{H \perp}=\left\|\vec{p}_{H \perp}\right\|$. 
With the explicit expression (65) for $M E$, this integral can be written more explicitly as

$$
\left.\frac{d^{2} \sigma}{d p_{H \perp}^{2} d \eta}\right|_{\eta=0}=\frac{1}{2 \pi^{3}}\left|C_{0} \mathcal{S}\left(\frac{s}{M^{2}}\right) f\right|^{2} \int_{0}^{\infty} d z z J_{0}\left(z p_{H \perp}\right)[h(z)]^{2},
$$

where

$$
\begin{gathered}
h(z)=\frac{\left(m_{1} m_{2}\right)^{4}}{\left(m_{2}^{2}-m_{1}^{2}\right)^{2}} \int_{0}^{\infty} d x x \int_{0}^{\frac{\pi}{2}} d \theta \operatorname{Re}\left[\exp \left(-\mathcal{S}\left(\frac{s_{1}}{M^{2}}\right) F\left(x_{+}^{2}\right)-\mathcal{S}^{*}\left(\frac{s_{1}}{M^{2}}\right) F\left(x_{-}^{2}\right)\right)\right] \\
\times\left[K_{0}\left(x_{+} m_{2}\right)-K_{0}\left(x_{+} m_{1}\right)\right]\left[K_{0}\left(x_{-} m_{2}\right)-K_{0}\left(x_{-} m_{1}\right)\right]
\end{gathered}
$$

with

$$
x_{ \pm}=\left\|\vec{x} \pm \frac{1}{2} \vec{z}\right\|=\sqrt{x^{2}+\frac{1}{4} z^{2} \pm x z \cos \theta} .
$$

The results of the numerical integration in (89) are presented in fig. 6 for $M=115 \mathrm{GeV} / c^{2}$. One immediately sees that the distribution peaks near $p_{H \perp}=0$ and that is has a width of the order of $1 \mathrm{GeV} / c$. This preference of the Higgs particle to be produced with a small momentum transfer should greatly facilitate its detection: it follows that its decays products have a transverse momenta that are balanced within approximately $1 \mathrm{GeV} / c$.

It turns out that these transverse-momentum distributions have almost identical shapes for the different values of the Higgs mass $M$. Therefore, for other values of $M$, this distribution can be obtained from the one of fig. 6 by merely scaling the curve with a factor

$$
\left.\frac{d \sigma(M)}{d \eta}\right|_{\eta=0} /\left.\frac{d \sigma(115)}{d \eta}\right|_{\eta=0}
$$

the values of which can be read off from fig. 5 .

The most prominent feature of the Higgs transverse distribution, shown in fig. 6, is that the peak at zero transverse momentum has a very narrow width of less than $1 \mathrm{GeV} / c^{2}$. Since this feature is of central importance in the detection of the Higgs particle for this class of events, as to be discussed in the next section, it is desirable to gain some intuitive feeling of this narrow width.

The first reason for expecting such a narrow width comes from the close relation between the production process (1) and proton-proton elastic scattering. This relation has been extensively used in developing the phenomenological model here, and has the implication that, both in elastic scattering and for process (1), the scale of the transverse momentum distributions is given by the mass of the proton.

A closely related second consideration concerns the size of the proton, which is of the order of 1 fermi. This leads to the transverse momentum of $0.2 \mathrm{GeV} / c$. For proton-proton elastic scattering, this is indeed roughly the scale for $d \sigma / d t$. This similar scale may be expected to be important for $d \sigma / d p_{H \perp}$ of Higgs production.

Such narrow width of the order of $1 \mathrm{GeV} / c$ is by no means limited to the specific Higgs production mechanism studied here, i.e., there are, for Higgs production at the Large Hadron Collider, other channels that also lead to such sharp peaks in the transverse distribution for the produced Higgs particle. This important point is discussed in more detail in ref. [23]. 
There are nevertheless significant differences between elastic scattering and Higgs production via gluon fusion. The following question is especially pertinent: Should this Higgs transverse distribution of fig. 6 be broadened significantly by the emission of one or more jets? This jet emission is not taken into account in the present phenomenology. The answer is that such broadening is expected to be unimportant due to the fact that emission of one jet is greatly suppressed. This suppression comes simply from color conservation, i.e., two Pomerons, one from each of the incident protons, cannot become a Higgs particle plus either a quark jet or a gluon jet. This point is to be discussed further in the next section.

It should also be noted that, in the present phenomenological model, no use is made of any gluon distribution function, in complete analogy with the phenomenology of elastic p-p scattering. This important point is later addressed in subsection 12.2.

\section{Comments on the Detection of the Higgs Particle}

As explained in the Introduction, the Higgs production process of interest here is (6), where most of the groups $A$ and $B$ of particles go down the two beam pipes. Since the particles that leak out of the beam pipes are expected to have relatively low energies, the events of process (6) are 'clean' in the sense that the observed particles are mostly from the decay of the Higgs boson. Such events are conveniently referred to as semi-exclusive.

Two of the most important characteristics of such events are:

(1) The number of particles observed in the detector is relatively small. This implies in particular that the combinatorial background is not a problem.

(2) The transverse momentum of the produced Higgs particle is of the order of $1 \mathrm{GeV} / c$. Such a small transverse momentum of the Higgs particle can be neglected in most cases. Therefore, there are two additional constraints for these semi-exclusive processes.

Because of these characteristics, the data analysis for these semi-exclusive events is necessarily different from the current methods being planned for the Large Hadron Collider. It is the purpose of this section to present a brief discussion of the detection of the Higgs particle in these semi-exclusive events. Of course, the actual data analysis for such events needs a great deal more work, involving Monte Carlo generation of the events, detector simulation, etc.

In the following five subsections, the Higgs decay modes $H \rightarrow Z Z, W^{+} W^{-}, b \bar{b}, \tau^{+} \tau^{-}$, and $\gamma \gamma$ are to be considered. These are all two-body decay modes, and they are arranged in order of decreasing masses for the decay products.

There are two general types of data analysis for Higgs events at the Large Hadron Collider. When there is a sufficient number of measurements from the decay products, the mass of the Higgs particle can be determined event-by-event. This is the first type of analysis and will be called Analysis (i). In the second type of analysis, to be called Analysis (ii), the mass of the Higgs particle is scanned over a suitable range. For each mass, the data sample is fitted using the available knowledge of the decay process. This procedure can be applied whether there is a sufficient number of measurements to pin down the Higgs mass event-by-event or not. In other words, this Analysis (ii) is based on having a significant sample of decay events from Higgs particles of the same mass. 
Subsection 10.1 is devoted to the decay $H \rightarrow Z Z$ and subsection 10.2 to $H \rightarrow W^{+} W^{-}$. In these two subsections, both $Z$ 's and both $W^{\prime}$ 's are assumed to be on mass shell. This does not mean that the decays $H \rightarrow Z Z^{*}$ and $H \rightarrow W W^{*}$, with $Z^{*}$ and $W^{*}$ off mass shell, are not important. On the contrary, these two decays are expected to be important for the detection of the Higgs particle at the Large Hadron Collider. They are not discussed in the present section because the on-shell analysis should be carried out before going on to the more complicated off-shell analysis. Clearly, for these on-shell cases, the mass of the Higgs particle must be larger than twice the mass of the $Z$ (subsection 10.1) or the $W$ (subsection 10.2).

One more comment is perhaps appropriate here. In some of the existing analyses for the detection of the Higgs particle, this Higgs particle is assumed to be produced in association with one or two jets, each of which being a quark jet or a gluon jet. The presence of these jets has been used to reduce background. In the present case of the semi-exclusive Higgs production process (6), the situation of the associated jets is different. Since the Pomeron has by definition no non-zero quantum number, it follows that

$$
\text { Pomeron }- \text { Pomeron }- \text { jet coupling }=0,
$$

for both quark jet and gluon jet. It is therefore not possible for the Higgs particle to be produced in association with one jet. It is however possible in association with two or more jets.

\section{1. $H \rightarrow Z Z$}

Consider first the case where both $Z$ 's decay leptonically. The branching ratios for these leptonic decay modes are, from p. 32 of ref. [24],

$$
\begin{aligned}
& Z \rightarrow e^{+} e^{-}, \quad(3.363 \pm 0.004) \%, \\
& Z \rightarrow \mu^{+} \mu^{-}, \quad(3.366 \pm 0.007) \% \text {, } \\
& Z \rightarrow \tau^{+} \tau^{-}, \quad(3.370 \pm 0.008) \%, \\
& Z \rightarrow \nu \bar{\nu}, \quad(20.00 \pm 0.06) \% \text {. }
\end{aligned}
$$

(a) The term 'golden channel' is often used to refer to the case where both $Z$ 's decay into $\ell^{+} \ell^{-}$, where $\ell$ means $e$ or $\mu$. The probability for this golden channel is about $0.45 \%$. This channel is described as golden because the momenta of all four particles in the final state $-4 e, 4 \mu$, or $2 e 2 \mu$ - can be accurately measured. For the semi-exclusive production of the Higgs particle [process (6)], this golden channel is not of great importance.

(b) Two other purely leptonic decay modes are $H \rightarrow Z Z$ followed by

$$
Z Z \rightarrow \ell^{+} \ell^{-} \tau^{+} \tau^{-}
$$

and

$$
Z Z \rightarrow \tau^{+} \tau^{-} \tau^{+} \tau^{-}
$$


Here, process (95) means that one of the two $Z$ 's decays into $\ell^{+} \ell^{-}$, while the other $Z$ decays into $\tau^{+} \tau^{-}$, and (96) means that both $Z$ 's decay into $\tau^{+} \tau^{-}$. These decay probabilities are $0.45 \%$ for $(95)$ and $0.11 \%$ for (96). For these two decay modes, the method of analysis for the case $H \rightarrow \tau^{+} \tau^{-}$can be applied; this is described in subsection 10.4. Using that method, it is found that, in both cases, Analysis (i) is applicable, and of course also Analysis (ii).

(c) A further leptonic decay mode is $H \rightarrow Z Z$ followed by

$$
Z Z \rightarrow \ell^{+} \ell^{-} \nu \bar{\nu}
$$

This is a good example for which the semi-exclusive process (6) is very well suited. The decay probability for the process (97) is about $2.7 \%$. It should also be noted that, since the Pomeron-Pomeron- $Z$ coupling vanishes, the observation of $Z$, e.g., through its decay into $\ell^{+} \ell^{-}$, must mean the presence of a second particle that is invisible. This second invisible particle is most likely a $Z \rightarrow \nu \bar{\nu}$.

Since this is the first example, the Analysis (ii) for this case is to be described in some detail. As usual, let $M$ be the mass of the Higgs particle: this is the variable to be scanned over a suitable range. For the purpose of determining the momentum of the $Z$ that decays into $\nu \bar{\nu}$ in terms of this mass $M$, let

$$
\left(p_{x}, p_{y}, p_{z}\right)=\left(\vec{p}_{\perp}, p_{z}\right)
$$

be the known momentum of the $Z$ that decays into $\ell^{+} \ell^{-}$. From characteristic (2) at the beginning of this section, the transverse momentum of the $Z$ that decays into $\nu \bar{\nu}$ is given by $-\vec{p}_{\perp}$. Thus, only the longitudinal momentum of this $Z$ is not known, but it can be expressed in terms of $M$. Actually, it is slightly more convenient to use the longitudinal component of the momentum of the Higgs particle; call it $x$. Then, it follows from energy conservation that

$$
\sqrt{M^{2}+x^{2}}=\sqrt{\bar{M}_{Z}^{2}+p_{z}^{2}}+\sqrt{\bar{M}_{Z}^{2}+\left(x-p_{z}\right)^{2}}
$$

where

$$
\bar{M}_{Z}=\sqrt{M_{Z}^{2}+\vec{p}_{\perp}^{2}}
$$

is the transverse mass of both Z's. Solving eq. (99) for $x$ yields

$$
x=\frac{M}{2\left(M_{Z}^{2}+\vec{p}_{\perp}^{2}\right)}\left\{p_{z} M \pm \sqrt{\left(M_{Z}^{2}+\vec{p}_{\perp}^{2}+p_{z}^{2}\right)\left[M^{2}-4\left(M_{Z}^{2}+\vec{p}_{\perp}^{2}\right)\right]}\right\} .
$$

Comparison of the observed distributions with the known properties of this decay mode gives the most likely value for the Higgs mass $M$.

This illustrates the general procedure of Analysis (ii), where a Higgs mass is assumed and eventually determined by a maximum likelihood or similar method, such as a neural network. Such a procedure can also be used for the cases (d) and (e) below. 
(d) Consider next the case where one of the two Z's decays hadronically, i.e.,

$$
Z \rightarrow h \bar{h} \text {. }
$$

while the other $Z$ decays leptonically:

$$
\begin{aligned}
& Z \rightarrow \ell^{+} \ell^{-}, \\
& Z \rightarrow \tau^{+} \tau^{-},
\end{aligned}
$$

or

$$
Z \rightarrow \nu \bar{\nu}
$$

These three cases are similar to the cases (a), (b), and (c) above, except that the $\ell^{+} \ell^{-}$ pair is replaced by the $h \bar{h}$ pair of (102). The channel of (103) with (102) is likely to be a useful one for the semi-exclusive production under discussion. The decay (104) with (102) shows similarity to (96) and can be analyzed in the same way. Since the measurement of the momentum of a hadron is in general less accurate than that of an electron or muon, the analysis of the decay (105) with (102) needs to be studied more carefully. On the other hand, this (105) with (102) does have the advantage of having a decay probability that is about ten times larger than the 'golden channel'.

(e) In the non-leptonic case, both of the two Z's decay hadronically through (102). In this case, the final state consists of four jets. At LEP II during the years 1999 to 2000, the process

$$
e^{+} e^{-} \rightarrow H Z \rightarrow \text { four jets }
$$

was studied carefully to search for the Higgs particle. Some of the techniques developed there can be used to analyze the non-leptonic case. Of course, there are also major differences: in the process (106), the energy and momentum in the center-of-mass system is accurately known, while this is not the case for the present non-leptonic case. Thus, a great deal more work needs to be carried out for (d) and (e).

It is worthwhile to summarize the reasons why the data analysis is possible for the semiexclusive production process (6). They are of course due to the two important characteristics of (6) given at the beginning of sec. 10. These semi-exclusive events from the Large Hadron Collider bear indeed a great deal of similarity to those from a high-energy electron-positron collider. Indeed, this is the first motivation given in the Introduction for the present study.

The first characteristic is the relatively small number of observed particles in the detector. This is what makes it possible to carry out the analysis of the various decay modes, especially for the cases (c), (d), and (e). Take for example case (e): in each event, there are just four jets in the final state due to pionization [25], and the purpose of the analysis is to determine whether these events with four jets are from the decay $H \rightarrow Z Z \rightarrow$ four jets. This relatively small number of observed particles already shows similarity to events from an electron-positron collider.

The second characteristic concerns the small transverse momentum of the produced Higgs particle, or, more generally, the total transverse momentum of the pionization product. With 
the semi-exclusive process (6) as due to the annihilation of two Pomerons to produce a Higgs, there is similarity to electron-positron annihilation but with the incoming leptons replaced by Pomerons. This Pomeron annihilation process is discussed in more detail in sec. 11 .

\section{2. $H \rightarrow W^{+} W^{-}$}

The important decay modes and the branching ratios for the $W$ are, from p. 31 of ref. [24]

$$
\begin{array}{ll}
W^{+} \rightarrow e^{+} \nu & (10.75 \pm 0.13) \%, \\
W^{+} \rightarrow \mu^{+} \nu & (10.57 \pm 0.15) \%, \\
W^{+} \rightarrow \tau^{+} \nu & (11.25 \pm 0.20) \% \\
W^{+} \rightarrow \text { hadrons } & (67.60 \pm 0.27) \%
\end{array}
$$

Remember, as discussed just before subsection 10.1, that the Higgs particle cannot be produced via the semi-exclusive process (6) with only one additional jet.

Following subsection 10.1, consider first the case where both the $W^{+}$and the $W^{-}$decay leptonically.

(a) Again, let $\ell$ denote $e$ or $\mu$. Then, from (107), the

$$
\text { branching ratio of } W^{+} \rightarrow \ell^{+} \nu \sim 21.32 \% \text {. }
$$

Thus, the decay probability for

$$
W^{+} \rightarrow \ell^{+} \nu, \quad W^{-} \rightarrow \ell^{-} \bar{\nu} .
$$

is about $4.5 \%$, which is ten times the 'golden channel' of subsection 10.1 .

In this final state, there are two leptons of opposite charge and two neutrinos. Even neglecting the transverse momentum of the produced Higgs particle, there are four independent unknown momentum components for the neutrinos, namely

$$
p_{\nu z}, p_{\bar{\nu} z}, p_{\nu x}, \text { and } p_{\nu y} \text {. }
$$

Since there are only two constraints, namely the masses of the $W^{+}$and the $W^{-}$, the kinematics is still not determined, even if a Higgs mass is assumed. Methods of data analysis such as maximum likelihood or neural networks are needed for this case. Note that the angular correlation between the observed $\ell^{+}$and $\ell^{-}$is part of the likelihood and is also taken into account for the neural network.

It is of interest to compare the present case with the (c) of the subsection 10.1 for $H \rightarrow Z Z$. In both cases, the final state consists of $\ell^{+}, \ell^{-}, \nu$, and $\bar{\nu}$. Yet, for that (c), the analysis can be carried through by scanning a range of Higgs masses, while this is not possible for the present case. The basic difference is that, for the previous (c), the $\nu$ and $\bar{\nu}$ form a $Z$. For each value of the Higgs mass being scanned, the momentum of this $Z$ is determined, but not the separate momenta of the $\nu$ and the $\bar{\nu}$. 
(b) Similar to the (b) of subsection 10.1, three other purely leptonic decay modes are $H \rightarrow W^{+} W^{-}$followed by

$$
\begin{array}{ll}
W^{+} \rightarrow \ell^{+} \nu, & W^{-} \rightarrow \tau^{-} \bar{\nu} ; \\
W^{+} \rightarrow \tau^{+} \nu, & W^{-} \rightarrow \ell^{-} \bar{\nu} ; \\
W^{+} \rightarrow \tau^{+} \nu, & W^{-} \rightarrow \tau^{-} \bar{\nu} .
\end{array}
$$

Because of the complication already encountered in the case (a), it is premature to study these (111), (112), and (113) before the decay (109) is understood.

(c) So far, both the $W^{+}$and the $W^{-}$decay leptonically. Consider next the case where one of the $W$ 's decays hadronically. From (107), the decay probability is larger than in the purely leptonic case. The first such case is

$$
W^{+} \rightarrow h h, \quad W^{-} \rightarrow \ell^{-} \bar{\nu}
$$

and its charge conjugate

$$
W^{+} \rightarrow \ell^{+} \nu, \quad W^{-} \rightarrow h h .
$$

If the basic idea of analyzing the decay $H \rightarrow \tau^{+} \tau^{-}$is applied to the hadrons here, the Analysis (ii) can be used.

(d) Similar to (114), there are also the decays

$$
W^{+} \rightarrow h h, \quad W^{-} \rightarrow \tau^{-} \bar{\nu}
$$

and its charge conjugate

$$
W^{+} \rightarrow \tau^{+} \nu, \quad W^{-} \rightarrow h h .
$$

The relation of (d) to (c) is similar to that of (b) to (a). In other words, the case (d) is more complicated than case (c), and it is better to have a good understanding of case $(\mathrm{c})$ before studying this case $(\mathrm{d})$.

(e) The hadronic decay of $H \rightarrow W^{+} W^{-}$followed by

$$
W^{+} \rightarrow h h, \quad W^{-} \rightarrow h h .
$$

is very similar to the corresponding case (e) of subsection 10.1. From (107), the decay probability for $(116)$ is quite large, being $(45.7 \pm 0.4) \%$. The comments at the end of subsection 10.1 apply here with little modification.

10.3. $H \rightarrow b \bar{b}$

In the previous two subsections 10.1 and 10.2, the decays of the Higgs particle into $Z Z$ and $W^{+} W^{-}$have been studied. Since the $Z$ and the $W^{ \pm}$are massive, being 91.2 and $80.4 \mathrm{GeV} / c^{2}$ respectively, the Higgs must be relatively heavy, over 182.4 and $160.8 \mathrm{GeV} / c^{2}$ respectively if the $Z$ and the $W^{ \pm}$are on shell. Furthermore, since the $Z$ and the $W^{ \pm}$decay 
rapidly, the decay products of the Higgs particle consist of at least four particles (including neutrinos).

In this and the next two subsections, the decays of the Higgs particle into $b \bar{b}, \tau^{+} \tau^{-}$, and $\gamma \gamma$ will be considered. There are two major differences between the previous two subsections and the next three. First, in these three subsections including the present one, the Higgs particle will be taken to be relatively light, so that the decays are relevant to the mass of $115 \mathrm{GeV} / c^{2}$, as indicated by the preliminary experimental evidence from the LEP accelerator $[18,19]$. Secondly, consider the case of $b \bar{b}$ for this subsection. Since the mass of the $b$ quark is only $4.2 \mathrm{GeV} / c^{2}$, it is seen in the experimental apparatus as a well-defined jet. This is even more true for the $\tau$, with the ' $\tau$-jet' consisting mostly of either one lepton or one or more hadrons. Thus, the final state due to Higgs decay can be considered to be two particles, either two quark jets, or two ' $\tau$-jets', or just two photons, to be contrasted with the four particles (including neutrinos) for the subsections 10.1 and 10.2.

When the produced Higgs particle decays into two particles, the smallness of the transverse momentum of the Higgs particle implies that these two particles are essentially backto-back in the transverse plane. This fact is of primary importance in all three cases 10.3, 10.4 , and 10.5 to be studied.

The details of the data analysis for these three cases are actually quite different. In the case of the $b \bar{b}$ mode for this subsection, only general remarks can be made, because the analysis depends sensitively on the capacity of the detector. In the next two subsection, the decays into $\tau^{+} \tau^{-}$and $\gamma \gamma$ will be studied; since they are less sensitive to the detector, they are to be discussed in more detail.

(1) For a Higgs mass near $115 \mathrm{GeV} / c^{2}$, this $H \rightarrow b \bar{b}$ is the major decay mode. Thus, this decay mode is of importance even if the detection efficiency is not high.

(2) One of the major problems in using this decay mode is how it can be triggered. In order to be sensitive to this mode, the trigger menu has to be arranged so that such events can be observed with reasonable efficiency.

(3) Since the leptonic branching ratio of the $b$ is about $20 \%$ including both electron and muon, it is acceptable to trigger on the leptonic decay of one of the $b$ 's.

(4) Since the decay under consideration here is

$$
H \rightarrow b \bar{b},
$$

$b$-jet identification is clearly desirable. For example, if the detector has this capability, then the observation of this decay mode, but not the corresponding ones into lighter quarks, gives strong indication that the particle seen couples more strongly to heavier quarks, and that it is likely to be the Higgs particle.

(5) It is, however, not clear how important it is to have this $b$-jet identification. Evidence that it is the Higgs particle of the standard model can come from other decay channels. Before the observed particle is identified as a Higgs particle, let us denote it by $X$. Evidence as to whether $X$ is the standard model Higgs can also come from the decay rate (117). Furthermore, it is not inconceivable that there may exist an unexpected 
particle $X$ that decays significantly into

$$
\begin{aligned}
& X \rightarrow u \bar{u}, d \bar{d}, \text { and/or } s \bar{s}, \\
& X \rightarrow c \bar{c} .
\end{aligned}
$$

The three channels of (118) are probably indistinguishable, and they may even be difficult to be distinguished from (119). If there is such a particle $X$, it would be interesting to know its spin.

10.4. $H \rightarrow \tau^{+} \tau^{-}$

This is an interesting decay mode, and it is to be used to illustrate the profound effect on its data analysis due to the smallness of the Higgs transverse momentum. See (2) at the beginning of the present section.

The $\tau$-lepton has many decay modes; the branching ratios for six of them are, from p. 34 of ref. [24]:

$$
\begin{array}{lll}
\tau^{-} \rightarrow \mu^{-} \bar{\nu}_{\mu} \nu_{\tau}, & (17.36 \pm 0.05) \%, & \\
\tau^{-} \rightarrow e^{-} \bar{\nu}_{e} \nu_{\tau}, & (17.85 \pm 0.05) \%, & \\
\tau^{-} \rightarrow \pi^{-} \nu_{\tau}, & (10.91 \pm 0.07) \%, & \\
\tau^{-} \rightarrow \pi^{-} \pi^{0} \nu_{\tau}, & (25.52 \pm 0.10) \%, & \\
\tau^{-} \rightarrow \pi^{-} \pi^{0} \pi^{0} \nu_{\tau}, & (9.27 \pm 0.12) \% & \text { exclude } K^{0}, \\
\tau^{-} \rightarrow \pi^{-} \pi^{+} \pi^{-} \nu_{\tau}, & (9.03 \pm 0.06) \% & \text { exclude } K^{0} .
\end{array}
$$

Taken together, they give $90 \%$ of the $\tau$ decay modes. The mass of $\tau$ is $m_{\tau}=1.777 \mathrm{GeV} / c^{2}$, which is about $1.5 \%$ of the $115 \mathrm{GeV} / c^{2}$ given before. Because of this $1.5 \%$ being quite small, a good approximation to the direction of $\tau$ is that of its visible decay products. As in the previous subsections, the small transverse momentum of the Higgs particle is neglected in the following analysis.

For definiteness, consider the first decay mode of (120), i.e.,

$$
H \rightarrow \tau^{+} \tau^{-}
$$

with

$$
\tau^{+} \rightarrow \mu^{+} \nu_{\mu} \bar{\nu}_{\tau} \quad \text { and } \quad \tau^{-} \rightarrow \mu^{-} \bar{\nu}_{\mu} \nu_{\tau}
$$

Let

$$
p_{\tau^{+}}, p_{\tau^{-}}, p_{\mu^{+}}, \text {and } p_{\mu^{-}}
$$

be respectively the four-momenta of the $\tau^{+}, \tau^{-}, \mu^{+}$, and $\mu^{-}$. Then, the approximations above mean that

$$
\begin{gathered}
p_{\tau^{+} x}+p_{\tau^{-} x}=p_{\tau^{+} y}+p_{\tau^{-} y}=0 \\
p_{\tau^{+}}=\left(\sqrt{m_{\tau}^{2}+\vec{p}_{\tau^{+}}^{2}}, p_{\tau^{+} x}, p_{\tau^{+} y}, p_{\tau^{+} z}\right) \sim\left(\left|\vec{p}_{\tau^{+}}\right|, p_{\tau^{+} x}, p_{\tau^{+} y}, p_{\tau^{+} z}\right), \\
p_{\tau^{-}}=\left(\sqrt{m_{\tau}^{2}+\vec{p}_{\tau^{-}}^{2}}, p_{\tau^{-} x}, p_{\tau^{-} y}, p_{\tau^{-} z}\right) \sim\left(\left|\vec{p}_{\tau^{-}}\right|, p_{\tau^{-} x}, p_{\tau^{-} y}, p_{\tau^{-} z}\right),
\end{gathered}
$$


and also that the are two positive numbers $\alpha_{+}$and $\alpha_{-}$such that

$$
\vec{p}_{\mu^{+}}=\alpha_{+} \vec{p}_{\tau^{+}} \quad \text { and } \quad \vec{p}_{\mu^{-}}=\alpha_{-} \vec{p}_{\tau^{-}}
$$

with

$$
0<\alpha_{+}<1 \text { and } 0<\alpha_{-}<1 .
$$

It follows from eqs. (124) and (125) that

$$
p_{\tau^{+}}=\frac{1}{\alpha_{+}} p_{\mu^{+}} \quad \text { and } \quad p_{\tau^{-}}=\frac{1}{\alpha_{-}} p_{\mu^{-}},
$$

and the substitution into eqs. (123) yields

$$
\frac{1}{\alpha_{+}} p_{\mu^{+} x}+\frac{1}{\alpha_{-}} p_{\mu^{-} x}=\frac{1}{\alpha_{+}} p_{\mu^{+} y}+\frac{1}{\alpha_{-}} p_{\mu^{-} y}=0,
$$

or

$$
\frac{p_{\mu^{+} x}}{p_{\mu^{-} x}}=\frac{p_{\mu^{+} y}}{p_{\mu^{-} y}}=-\frac{\alpha_{+}}{\alpha_{-}} .
$$

It is convenient to define this ratio as $r$, i.e.,

$$
r=\frac{\alpha_{+}}{\alpha_{-}} .
$$

Thus, the value of this ratio $r$ is immediately obtained from the measured momenta of the $\mu^{+}$and $\mu^{-}$, although not $\alpha_{+}$nor $\alpha_{-}$separately. It also follows from eqs. (128) that the four-momentum of the Higgs particle is given by

$$
p_{H}=p_{\tau^{+}}+p_{\tau^{-}}=\left(\frac{\left|\vec{p}_{\mu^{+}}\right|}{\alpha_{+}}+\frac{\left|\vec{p}_{\mu^{-}}\right|}{\alpha_{-}}, 0,0, \frac{p_{\mu^{+} z}}{\alpha_{+}}+\frac{p_{\mu^{-} z}}{\alpha_{-}}\right) .
$$

The Lorentz transformation to the rest frame of the Higgs particle is given by a velocity $\beta$ along the $z$-direction, where

$$
\tanh \beta=\frac{\frac{1}{\alpha_{+}} p_{\mu^{+} z}+\frac{1}{\alpha_{-}} p_{\mu^{-} z}}{\frac{1}{\alpha_{+}}\left|\vec{p}_{\mu^{+}}\right|+\frac{1}{\alpha_{-}}\left|\vec{p}_{\mu^{-}}\right|},
$$

or, by the definition (130),

$$
\tanh \beta=\frac{p_{\mu^{+} z}+r p_{\mu^{-} z}}{\left|\vec{p}_{\mu^{+}}\right|+r\left|\vec{p}_{\mu^{-}}\right|}
$$

The important point here is that every quantity on the right-hand side of eq. (132) is known, i.e., the Lorentz transformation to the rest frame of the produced Higgs particle is given by the measured momenta of the $\mu^{+}$and the $\mu^{-}$.

With this Lorentz transformation, the directions of the $\tau^{+}$and the $\tau^{-}$in the rest frame of the Higgs particle are known. They are, of course, back-to-back. This information can be 
used in various ways. First, the distribution of these directions tells immediately whether this decay $H \rightarrow \tau^{+} \tau^{-}$is consistent with the spin of $H$ being 0 . Secondly, for the decays (122) with high energy $\tau^{+}$and $\tau^{-}$, the distributions of $\alpha_{+}$and $\alpha_{-}$, as defined by eqs. (126), are well-known, and therefore also that of $r$, as given by eq. (130). Actually, a more symmetrical variable is, for example,

$$
\theta=2 \arctan r-\frac{\pi}{2},
$$

which ranges from $-\frac{\pi}{2}$ to $\frac{\pi}{2}$. The variable $\theta$ has the nice property that $\theta \rightarrow-\theta$ when $\alpha_{+}$and $\alpha_{-}$are exchanged. Of course, $\alpha_{+}, \alpha_{-}, r$, and $\theta$ all do not change under this Lorentz transformation. Thus, a second check is to see whether the experimental data give a distribution that is even in $\theta$.

Thirdly, and perhaps more interestingly, the distribution of $\alpha_{+}$and $\alpha_{-}$can be used to give a determination of the Higgs mass. This is an especially simple case of Analysis (ii).

Although the above discussion in this subsection has been given for the case where both $\tau$ 's decay through the first mode of (120), this analysis can be trivially extended to the other five modes of (120). For the second decay mode, it is only necessary to replace the $\mu$ 's by $e$ 's. For the four hadronic modes, the $\mu$ 's are to be replaced respectively by

$$
\begin{array}{ll}
\pi^{-} & \text {one charged track from } \pi^{-}, \text {no } \pi^{0}, \\
\pi^{-} \pi^{0} & \text { one charged track from } \pi^{-}, \text {one } \pi^{0}, \\
\pi^{-} \pi^{0} \pi^{0} & \text { one charged track from } \pi^{-}, \text {two } \pi^{0} \text { s, } \\
\pi^{-} \pi^{+} \pi^{-} & \text {three charged tracks from } \pi^{ \pm}, \text {no } \pi^{0} .
\end{array}
$$

Using the list (120), there are six such cases where the $\tau^{+}$and $\tau^{-}$decay similarly. There are also fifteen other decay combinations where the $\tau^{+}$and $\tau^{-}$decay through different modes of (120); charge conjugate decays have been considered to be the same. The above analysis can also be applied here, with small changes, to these fifteen cases.

The data analysis for this $\tau^{+} \tau^{-}$case does depend on the capability of the detector at the Large Hadron Collider. While there is no difficulty in separating the leptonic decay modes of the $\tau$ from the hadronic decay modes, it is less clear whether the four hadronic decay modes of (120) can be separated from each other, i.e., whether the four cases (134a)-(134d) can be identified. There is no problem in separating (134d) from the other three, since, among these four, (134d) is the only one with three charged tracks. Separating (134a), (134b), and (134c) depends on separating $\pi^{-}$from $\pi^{-} \pi^{0}$ if the $\pi^{0}$ hit in the electromagnetic calorimeter is close to that of $\pi^{-}$, and also the ability of telling one $\pi^{0}$ from two $\pi^{0}$ 's.

[For this application, the capability of the detector to separate $\pi^{ \pm}$from $K^{ \pm}$is not crucial. The reason is that $\tau$ rarely decays into a $K$. For example, the ratio

$$
\frac{\text { braching ratio of } \tau^{-} \rightarrow K^{-} \nu_{\tau}}{\text { braching ratio of } \tau^{-} \rightarrow \pi^{-} \nu_{\tau}} \sim 6 \%,
$$

and the corresponding ratio with an additional $\pi^{0}$ is even smaller.]

If the detector works well, two more modes in addition to the six of (120) may be included:

$$
\begin{array}{ll}
\tau^{-} \rightarrow \pi^{-} \pi^{+} \pi^{-} \pi^{0} \nu_{\tau}, & (4.48 \pm 0.06) \%, \text { exclude } K^{0}, \\
\tau^{-} \rightarrow \pi^{-} \pi^{0} \pi^{0} \pi^{0} \nu_{\tau}, & (1.04 \pm 0.07) \%, \text { exclude } K^{0} .
\end{array}
$$




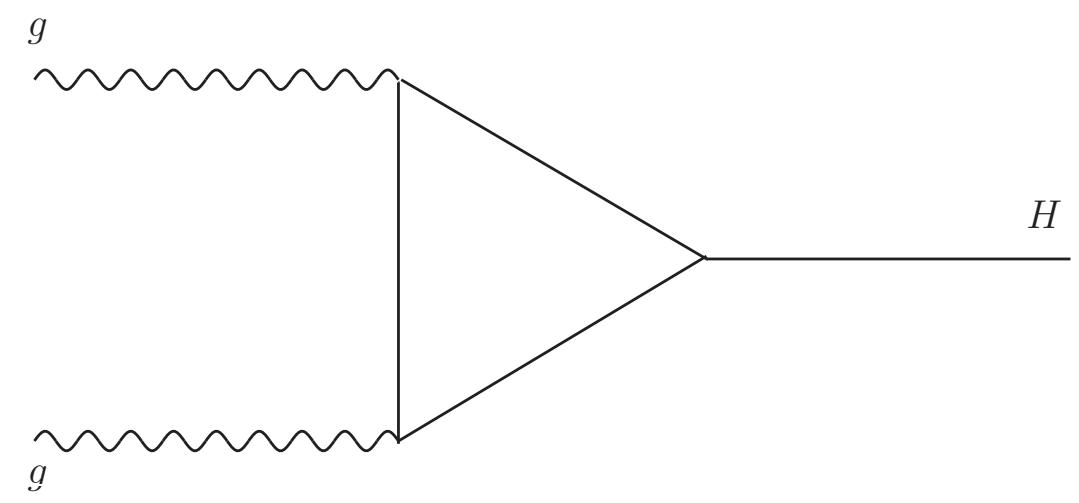

Figure 7: The diagram for Higgs production by gluon fusion. In the standard model, the particle in the triangular loop is the top quark.

Furthermore, if $\gamma$ can be clearly seen, then $\tau^{-} \rightarrow e^{-} \bar{\nu}_{e} \nu_{\tau} \gamma$ may also be included.

\section{5. $H \rightarrow \gamma \gamma$}

This decay mode is most interesting and has surprising and possibly far-reaching features. Unlike the previous four subsections, the discussion here applies not only to the semi-exclusive process (6) but also equally well to the inclusive production process

$$
p+p \rightarrow H+\text { anything }
$$

The field-theoretic development of the present theory and phenomenology, as described in ref. [5], is closely related to the process of gluon fusion shown in fig. 7. Since the Higgs particle does not couple directly to the massless gluon, this gluon fusion process can only proceed through a triangular loop of a heavy particle, which is the top quark in the standard model. Other particles can also contribute through this loop, but that from the top quark dominates.

If this diagram of fig. 7 is turned around and the gluons are reinterpreted as photons, then the result is that of fig. 8: the decay of the Higgs particle into two photons.

Once again, in the standard model, an important contribution to this $\gamma \gamma$ decay is from the top quark triangle (besides other contributions like a $W$ triangle).

The situation is much more interesting if the standard model is not the whole story. In that case, there may well be additional heavy particles besides the top quark that contribute significantly to these triangles. In general, the list of new particles that give such significant contributions may not be the same for the diagrams of fig. 7 and fig. 8: for the triangle of fig. 7, the particle must have color so that it couples to the gluons; and, for the triangle of fig. 8, the particle must have an electric charge so that it couples to the photon. If the contributing heavy particle is spin-0, then the triangle is, of course, not the only diagram.

This situation bears some resemblance to that of the LEP Collider in 1989. The first major experimental result from LEP was the determination of the number of generations 


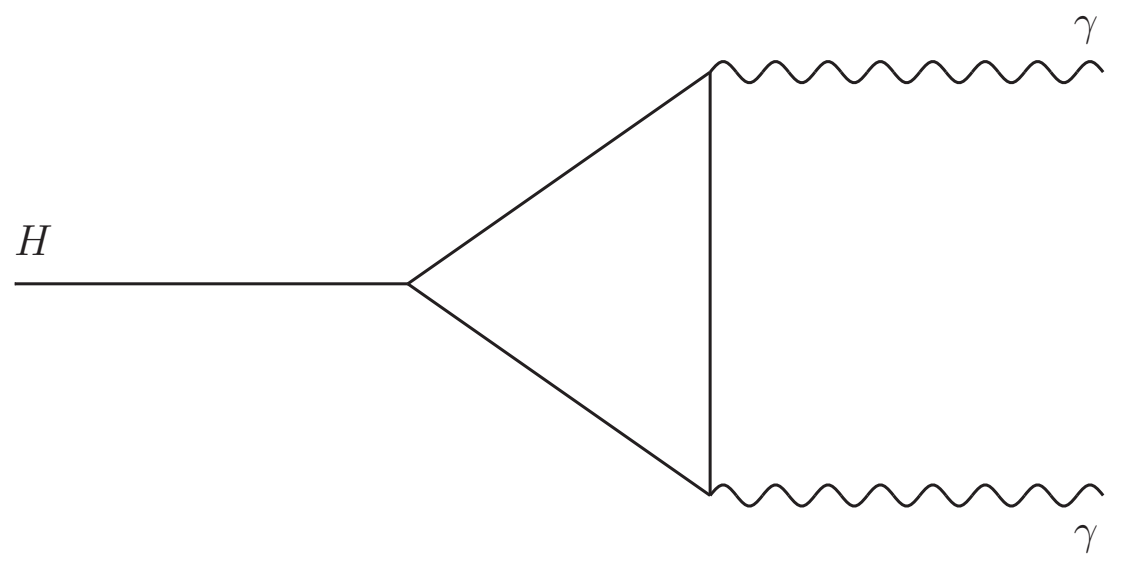

Figure 8: The diagram for the Higgs decay $H \rightarrow \gamma \gamma$. Again in the standard model, the particle in the triangular loop is the top quark.

with light neutrinos from the width of the $Z$. The number of light neutrino species, and hence the number of such generations, was found to be 3 [26]. This was accomplished without observing any neutrino at all.

In the present case, from the production cross section of the Higgs particle, or more precisely from the decay of the Higgs particle into two photons, information can be obtained about the possible existence of new heavy particles. In the case of the decay into two photons, the rate may be normalized to any decay process that does not depend on the triangular loop, one example being the leptonic decay of subsection 10.4:

$$
H \rightarrow \tau^{+} \tau^{-} \text {. }
$$

Similar to the LEP case, information about these heavy particles can be obtained without directly observing them in the experiment. Because of this exciting possibility, the triangular loop in fig. 7 and fig. 8 has been sometimes referred to as the 'magic triangle'.

There is a major difference between the triangle of fig. 7 and that of fig. 8. Additional contributions to fig. 7 change the rate for the production process (6), and such changes may be difficult to ascertain unless they are quite large. On the contrary, additional contributions to fig. 8 can be seen, even if not large, by observing a ratio such as $H \rightarrow \gamma \gamma / H \rightarrow \tau^{+} \tau^{-}$. Therefore, this or similar ratios are more sensitive to heavy charged particles, supersymmetric or not, that couple to the Higgs being studied. In the special case of supersymmetry, the use of the 'magic triangle' sidesteps the problem of dealing with the huge parameter space consisting of more than one hundred independent dimensions, and hence, the need of restricting these parameters.

\section{LHC as a Pomeron Collider}

In the Introduction, two motivations or purposes have been given for the present investigation. The second purpose, to generalize the basic idea that leads to the increasing total 


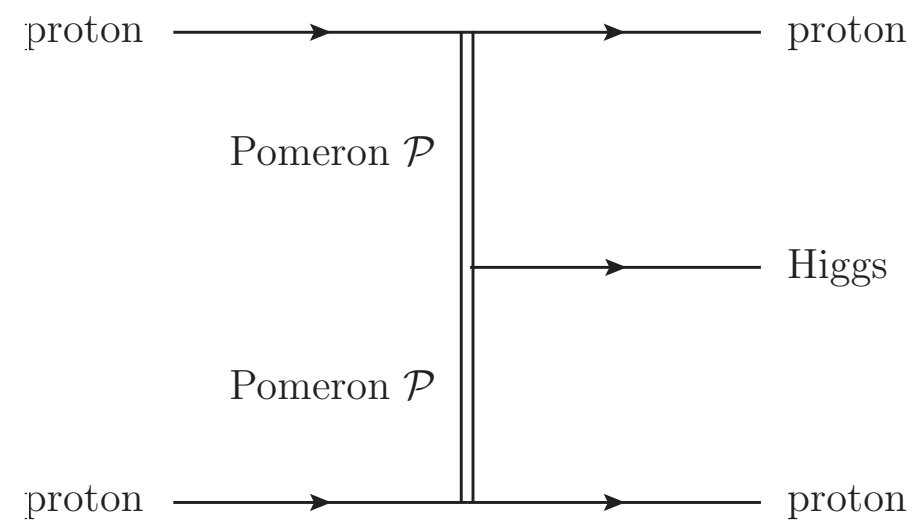

Figure 9: Schematic representation of the Higgs production process $p+p \rightarrow p+H+p$ by pionization [25].

cross section to production processes, has largely been fulfilled in the prediction of the cross sections for Higgs production at the Large Hadron Collider. This section is devoted to the first purpose.

This first purpose consists of two closely related parts: to look for a subset of LHC events that are 'clean', and to ask whether this subset of relatively clean events can provide information similar to those expected from a $\mathrm{TeV}$ electron-positron linear collider. So far in this paper, it has been seen that the Higgs production processes (1) and (6) do provide 'clean' events. It remains to determine further cases of such 'clean' events and to compare them with the $e^{+} e^{-}$events.

For the phenomenology developed here, the process (1) is described by that of fig. 9 see also ref. [5]. In this fig. 9, each of the incident protons emits a Pomeron, and the two Pomerons combine to produce the Higgs $H$. Therefore, the core process for both (1) and (6), is this annihilation of these two Pomerons as shown in fig. 10:

$$
\text { Pomeron }+ \text { Pomeron } \rightarrow H \text {. }
$$

The annihilation of the two Pomerons can lead not only to a Higgs boson, but more generally to a variety of final states, also shown in fig. 10:

$$
\text { Pomeron }+ \text { Pomeron } \rightarrow X
$$

where $X$ may consist of a single particle or more than one particle. Viewed in this way, there is clearly a great deal of similarity between this (140) and electron-positron annihilation, $e^{+} e^{-} \rightarrow Y$.

Because of this process (140), the Large Hadron Collider may be considered to be a Pomeron collider. [Of course, (140) describes only a small fraction of the processes at the Large Hadron Collider.] The systematic study of the large number of such processes is a very major job, and the complexity is expected to be comparable to the many processes at a $\mathrm{TeV}$ electron-positron linear collider. The purpose of this section is to give a brief introduction 


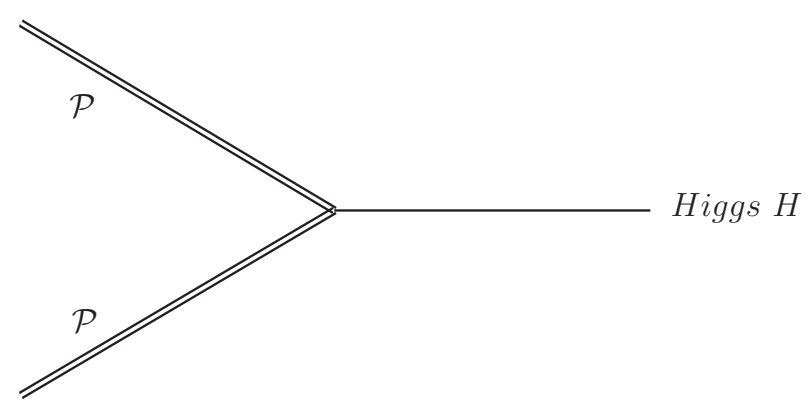

(a)

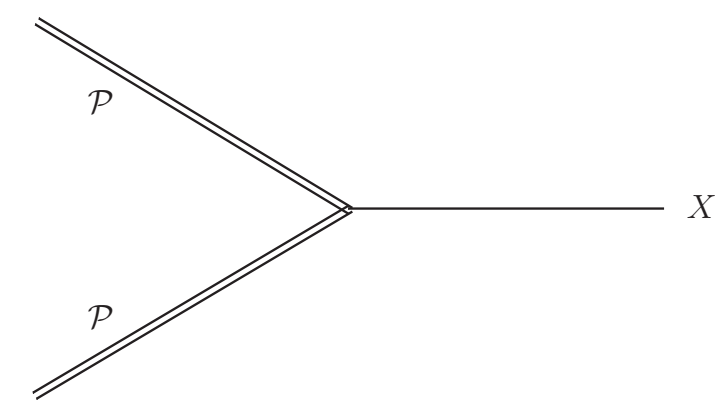

(b)

Figure 10: Annihilation of two Pomerons into (a) $H$, process (139), and (b) $X$, process (140).

to this vast area of future research and to show some of the similarities and differences to an $e^{+} e^{-}$collider.

It may be argued that, for the $e^{+} e^{-}$annihilation, not only the transverse momentum, but also the longitudinal momentum of the $e^{+} e^{-}$system is zero. This is indeed true for a linear electron-positron collider with a center-of-mass energy of $0.5 \mathrm{TeV}$ or less; at higher energies, when beamstrahlung becomes important [27], this longitudinal momentum also varies from event to event. Thus, at such higher energies, the similarity is even more striking.

\subsection{Triple Higgs coupling}

The study of Higgs triple coupling is one of the important physics topics for the proposed $\mathrm{TeV}$ electron-positron collider. The relevant process is not the annihilation of the electron and the positron, but

$$
e^{+}+e^{-} \rightarrow \bar{\nu}_{e}+H+H+\nu_{e},
$$

and the diagrams are those shown in fig. 11 [28]. Note that the $H H H$ coupling appears in Fig 11(c).

To study the same Higgs triple coupling at the Large Hadron Collider, a second Higgs 


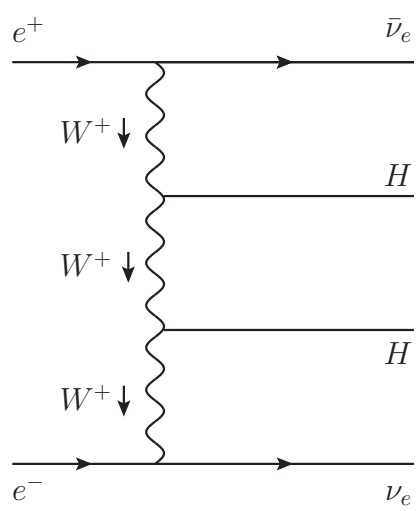

(a)

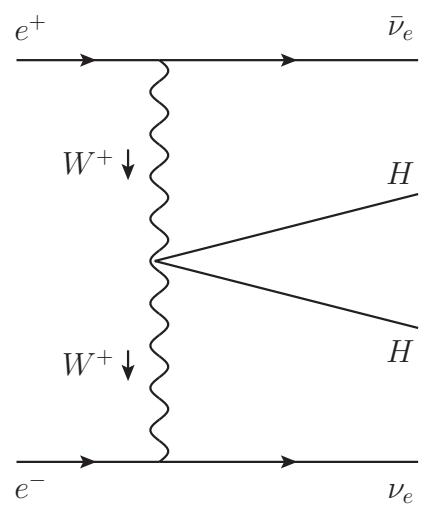

(b)

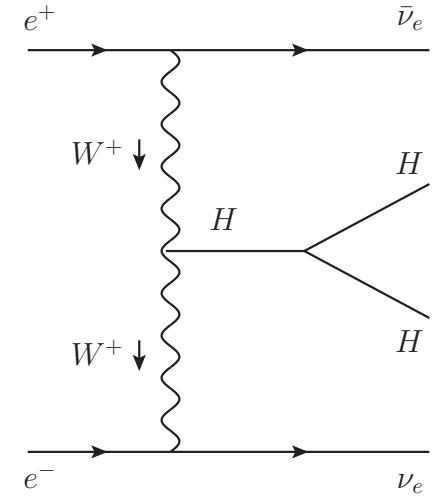

(c)

Figure 11: The diagrams for the process (141) for the study of the triple Higgs coupling at a TeV electronpositron linear collider.

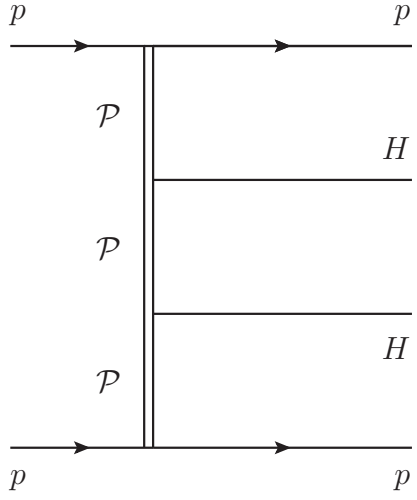

(a)

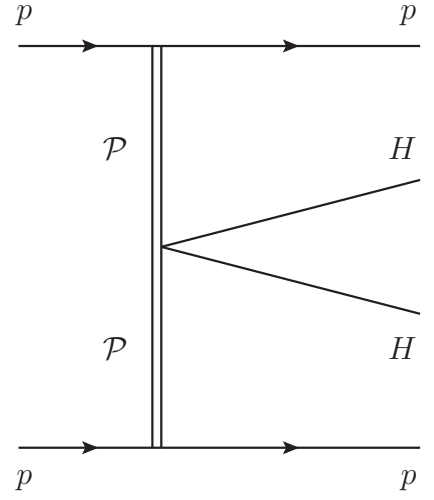

(b)

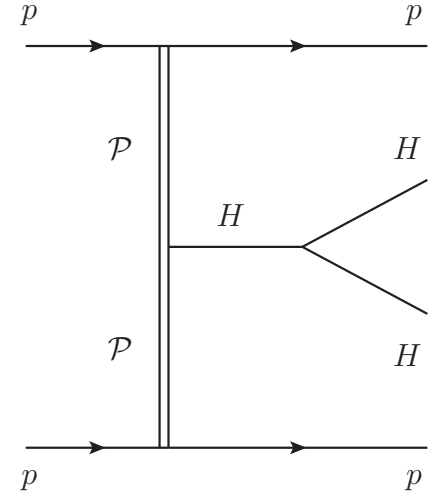

(c)

Figure 12: The diagrams for the process (142) for the study of the triple Higgs coupling at the Large Hadron Collider.

boson is added to the process (1), leading to

$$
p+p \rightarrow p+H+H+p .
$$

Following fig. 9, the diagrams for this process (142) are those of fig. 12. Again, the $H H H$ coupling appears in fig. 12(c). If these three diagrams of fig. 12 were drawn in more detail, the one top triangle would appear fig. 12(c), two top triangles in fig. 12(a), while one top square would appear in fig. 12(b). The discussion in subsection 10.5 applies not only to the top triangle, but equally well to the top square that gives the $g g H H$ coupling. 
A comparison of fig. 11 and fig. 12 shows striking similarities in these two ways of studying the triple Higgs coupling, at the proposed electron-positron linear collider and at the Large Hadron Collider.

Following the consideration in the Introduction, the actual process to be studied at the LHC is not (142), but

$$
p+p \rightarrow A+H+H+B
$$

similar to (6), where most of the group $A$ of particles go down one beam pipe, and those of group $B$ go down the other beam pipe. In this (143), the two Higgs bosons $H$, or rather their decay products, constitute the event signature. In this way, this semi-exclusive process (143) also leads to 'clean' events at the LHC.

\subsection{Some properties of the produced states}

In the above example of studying the triple Higgs coupling, the $\mathrm{TeV}$ electron-positron linear collider actually functions as a $W^{+} W^{-}$collider. Thus, the Pomerons from LHC play the roles of the $W$ 's. In many other cases, the electron and the positron annihilate each other to produce interesting final states; in these cases, as seen from (140), the Pomerons from LHC play the role of the electron and the positron. Here is a brief discussion of such cases.

The major difference is this: while the electron-positron annihilation leads to a state which is odd under charge conjugation, the Pomeron annihilation gives a state which is even. For electron-positron annihilation, the even states are reached by radiative decay; entirely similarly, for Pomeron annihilation, the odd states are reached through radiative decay. In this way, both even and odd states can be reached in both cases.

In summary, the LHC as a Pomeron collider may be advantageous in producing charge conjugate even states. In as much as no $\mathrm{TeV}$ electron-positron linear collider is likely to be built in the next decade, it may be interesting to use the LHC to reach directly the charge conjugate odd states through the process

$$
\text { Pomeron }+ \text { Pomeron } \rightarrow X^{\prime}+\gamma \text {. }
$$

In particular, it is important to develop methods to calculate theoretically the cross sections for both the processes (140) and (144).

[As discussed in ref. [5], the use of the word 'Pomeron' is slightly misleading. What each proton emits is not a Pomeron $\mathcal{P}$ but instead a $\mathcal{Q}$ as described in that reference. This $\mathcal{Q}$ is related to, but not the same as, $\mathcal{P}$.]

We conclude this sec. 11 with the following two observations. First, the design luminosity of the Large Hadron Collider, $10^{34} \mathrm{~cm}^{-2} \mathrm{~s}^{-1}$, is exceptionally high for a proton collider. Secondly, up to half a TeV, which is the highest center-of-mass energy of the $e^{+} e^{-}$linear collider listed in ref. [24], the cross section for Pomeron-Pomeron annihilation process (140) does not change much. For both of these two reasons, the Large Hadron Collider is an excellent accelerator as a Pomeron Collider. 


\section{Conclusion and Discussions}

Since the present paper treats several related but distinct topics, it may be useful to provide a summary.

First, on the basis of the field-theoretic results of ref. [5] and the knowledge about protonproton elastic scattering at high energies [see sec. 2], a phenomenological model is developed for the Higgs production process (6). As already emphasized, in this particular process of interest, most of the group $A$ of particles go down one beam pipe, while those of the group $B$ go down the other beam pipe. This process (6) thus gives only a fraction of the cross section for producing the Higgs boson at the Large Hadron Collider. This phenomenological model is given in secs. 3-8.

Secondly, this phenomenological model is used to give a theoretical prediction for the cross section of this class of Higgs production processes at the LHC. This numerical calculation is presented in sec. 9.

This class of events has several desirable features. The events are 'clean' in the sense that the events from electron-positron colliders are considered to be clean. Furthermore, the Higgs boson produced in this way has little transverse momentum, of the order of $1 \mathrm{GeV} / c$. These features imply that the resulting experimental data need to be analyzed in a different way. This new data analysis is given in sec. 10 .

Fourthly, this class of interesting events at the Large Hadron Collider can be readily generalized by replacing the Higgs boson by various states, either known ones or those that remain to be discovered:

$$
\text { proton }+ \text { proton } \rightarrow A+X+B \text {. }
$$

With this (145) rewritten in the form of (140), the LHC functions as a Pomeron collider. Through these 'clean' events, the Large Hadron Collider can give information similar to those expected from a TeV electron-positron linear collider, which remains to be built in the future. Sec. 11 gives a brief discussion of this vast area for future research.

In principle, a good test of these ideas could be dijet production at the Tevatron or the Large Hadron Collider. However, for the present model, the conditions (5) or (14) are of essential importance, and it is not obvious how to generalize those conditions to the case of dijet production. Along the same lines, one could think of single diffractive $Z$ production, but that process cannot be described in our model because there is no Pomeron-Pomeron- $Z$ vertex. The same conclusion holds for single diffractive $W$ production.

From this summary, it is seen that the following two topics deserve a more detailed discussion.

\subsection{Phenomenological model}

The most important point to be discussed is: why is it possible to develop a phenomenology, such as the one presented in secs. 3-8, without any experimental data for Higgs production at any energy through proton-proton interaction? This is to be contrasted with the phenomenology for proton-proton elastic scattering, where the data from $p$ - $p$ interactions at lower energy play an essential role. 


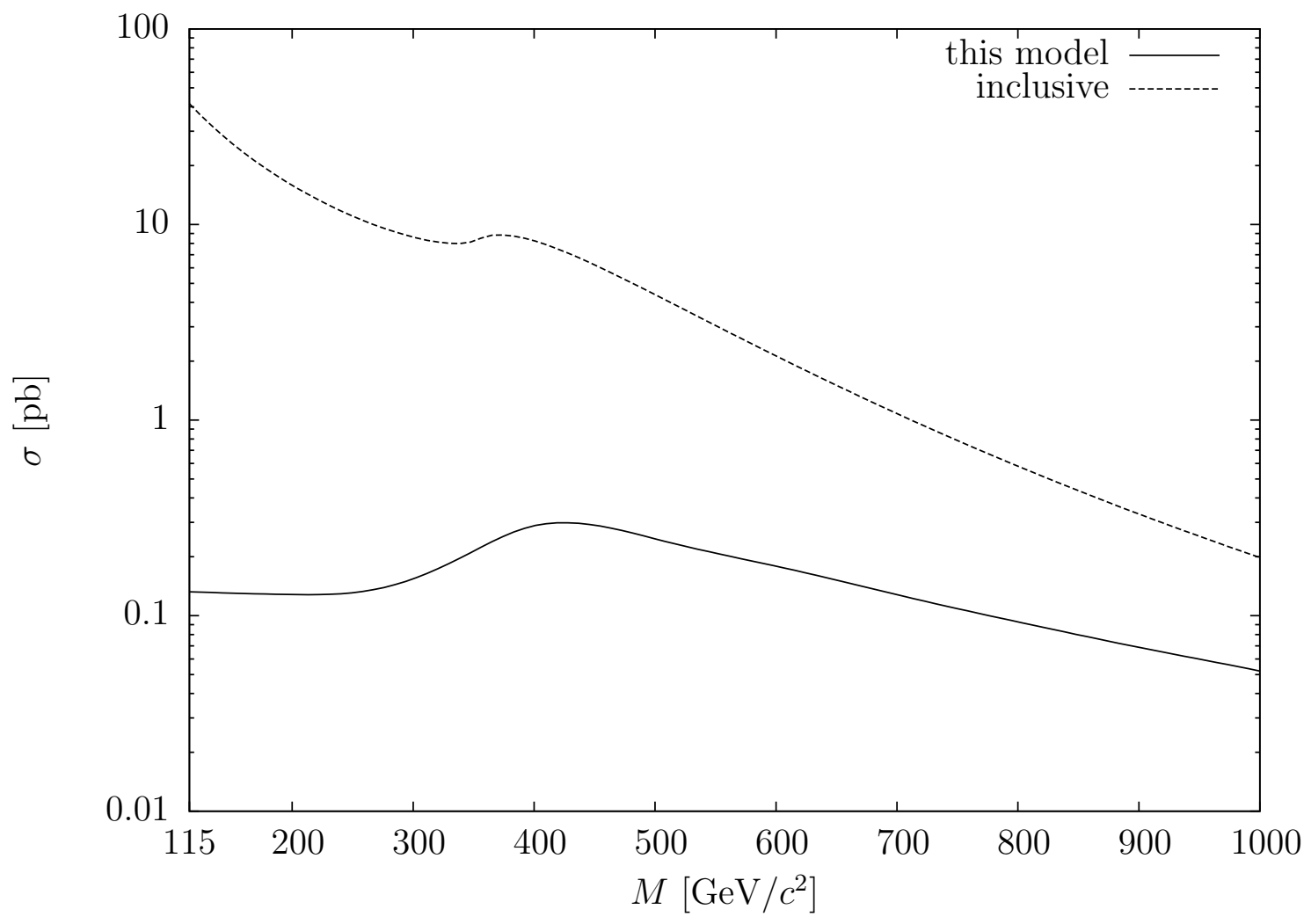

Figure 13: Comparison of the Higgs production cross sections for the processes (6) and (146).

(1) In order to develop the present phenomenology, information must be incorporated about the structure of the proton, which is necessarily very complicated. This is the underlying reason why, for the phenomenology of $p$ - $p$ elastic scattering at high energies, low energy $p-p$ data are needed. For the present paper, this problem is solved in the following way: instead of the low energy Higgs production data (which do not exist), information about the proton structure is incorporated by using the previously developed phenomenology for proton-proton scattering — see sec. 2.

(2) In addition to the proton structure, it is also necessary to have experimental information about the Pomeron-Pomeron-Higgs coupling. Since this information does not exist, this problem is more difficult to deal with. This issue has been referred to in sec. $3 \mathrm{ff}$; here is a more explicit discussion.

The appearance of $\mathcal{S}\left(s / m^{2}\right)$ as given by eq. (9) implies that the Pomeron is related to a fixed Regge cut. The underlying reason is that four-dimensional gauge field theories are renormalizable but not super-renormalizable [29]. With reference to (21f), let $\mu_{0}$ be that least upper bound of the spectrum of the operator $\mathcal{K}_{t}$, and $\phi_{0}$ the (improper) eigenvector corresponding to this $\mu_{0}$. In order to go from the $\mathcal{M}_{T}$ of (21f) to the $I$ of $(27 \mathrm{f})$, information about this $\phi_{0}$ is needed. This information we do not have for the proton. 
(3) Since attempts to get even a rough approximation to this $\phi_{0}$ for the proton have failed, it is necessary to seek an alternative approach. The following fact is what saved us: when the produced Higgs boson has no transverse momentum, a good approximation to the production amplitude can be written down without using any information about the Pomeron-Pomeron-Higgs coupling.

(4) In order to have a useful phenomenology for this class (6) of Higgs production at the Large Hadron Collider, it is essential to be able to extend to the case where the produced Higgs has a non-negligible transverse momentum. This extension has been carried out in sec. 6 using Hölder's inequality.

(5) In carrying out this extension, the obvious condition has been imposed that there should be no discontinuity as the transverse momentum of the produced Higgs goes to zero - this is eq. (41p). Moreover, the simplest way is chosen to fulfill this condition (41p). However, it turns out, as perhaps to be expected, that satisfying this condition restricts the validity of the phenomenological model to this transverse momentum not being too large. The region of validity depends not only on the mass but also on the rapidity of the Higgs boson in the proton-proton center-of-mass system.

(6) The phenomenological model developed in secs. 3-8 may be described succinctly as follows. On the basis of the theoretical considerations combined with the knowledge of proton-proton elastic scattering at high energies, a first phenomenology has been developed successfully. This first phenomenology has important limitations and remains to be improved using the forthcoming experimental data from the Large Hadron Collider. More precisely, the data from the LHC should give us information about the Pomeron-Pomeron-Higgs coupling, and this information is to be used to make major improvements upon this first phenomenology.

\subsection{Comparison of cross sections}

It is interesting and informative to compare the present cross sections for the process (6) with the usual cross section for the inclusive Higgs production [30]

$$
p+p \rightarrow H+X
$$

This comparison is shown in fig. 13.

(1) It is readily seen from this fig. 13 that, while the inclusive cross section for (146) decreases rapidly with increasing Higgs mass, that for (6) remains relatively constant.

(2) At low Higgs masses, the inclusive cross section is much larger than that for (6). On the other hand, for large Higgs masses, these two cross sections become comparable. This raises the following question: is the inclusive cross section truly inclusive? More specifically, the issue is whether this usual inclusive cross section properly contains that for process (6). As shall be emphasized under (4), caution is needed in making this comparison because some approximations are made in the present model. It is hoped that, in the future when data on Higgs production become available, these approximations can be improved or even circumvented. 
(3) For some calculations of Higgs production in hadron collisions, the single parton distribution functions constitute an essential ingredient. They are almost entirely obtained from HERA data and their use in hadron interactions is not always justified, like in the present model where the two gluons in the Pomeron are highly correlated. Perhaps a more sophisticated framework using parton correlation functions [31] can be used for the kind of final state that is considered here, but in the present model there is no need for them.

In the usual treatments of Higgs production [7, 8, 9], one can introduce a Sudakov form factor [32] to suppresses gluon emission in the rapidity gaps. However, in the successful treatment of elastic $p-p$ scattering $[12,13]$, no such Sudakov factor was ever introduced. Because the present model for Higgs production is firmly based on the elastic scattering phenomenology, the suppression of gluon bremsstrahlung is also inherent to the Higgs production model and it would be erroneous to apply a Sudakov factor in addition. The absence of the Sudakov form factors is another distinguishing feature of the present model, besides the ones listed in the Introduction.

(4) It should be pointed out that the curve for the cross section of (6) given in fig. 13 cannot be considered to be highly accurate, i.e., much less accurate than the numerical results of fig. 5. The question here is: how does one get this cross section from the rapidity distribution $d \sigma / d \eta$ as predicted by the phenomenology?

The procedure used is as follows. As discussed in detail under (5) of subsection 12.1 and seen explicitly in fig. 5 , this predicted $d \sigma / d \eta$ has only a limited range of validity in $\eta$, especially for the higher values of the Higgs mass. Also from this figure, in this limited range of $\eta$, the $d \sigma / d \eta$ does not depend much on the value of $\eta$. The approximation is therefore made that $d \sigma / d \eta$ is independent of $\eta$ in this limited range of $\eta$.

Pushing this approximation even further, this value of $d \sigma / d \eta$ independent of $\eta$ is to be used not only in this limited range of $\eta$ but for all $-2.5<\eta<2.5$ [see sec. 9]. With this less accurate approximation, the cross section shown in fig. 13 for (6) is obtained by integrating this $d \sigma / d \eta$ over this range of $\eta$, i.e., this cross section for (6) is given approximately by

$$
\sigma=\left.5 \frac{d \sigma}{d \eta}\right|_{\eta=0} .
$$

The choice of this factor of 5 is of course fairly arbitrary. Furthermore, this factor should depend on the Higgs mass. Since there seems to be no simple way to determine this dependence, eq. (147) is used for the entire range of Higgs mass.

(5) If the answer to the question raised under the above (2) is no, then the implication is that not all gluon fusion processes can be described in terms of the gluon distribution function for the proton. A strong indication that this is indeed the case is provided by the fact that, for the present pionization process, the Higgs particle cannot be produced in association with one jet, as discussed just before subsection 10.1. It should be emphasized that, in the entire development presented in ref. [5] and the present paper, no use is made of any parton distribution function. 


\section{Acknowledgements}

For the most helpful discussions, we are greatly indebted to Professors Claude Bourrely, Igor Ivanov, André Martin, Jan Perz, Jacques Soffer, and Bryan Webber.

\section{References}

[1] H. Cheng and T.T. Wu, Phys. Rev. Lett. 24 (1970) 1456.

[2] H. Cheng, J.K. Walker, and T.T. Wu, Phys. Lett. B 44 (1973) 97; U. Amaldi, et al., Phys. Lett. B 44 (1973) 112; S.R. Amendolia, et al., Phys. Lett. B 44 (1973) 119.

[3] Y. Dokshitzer, V. Khoze, and S. Troyan, in Physics in Collision VI, Proceedings of the Intenational Conference, Chicago, Illinois, 1986, edited by M. Derrick (World Scientific, Singapore, 1987), p. 365; J.D. Bjorken, Phys. Rev. D 47 (1993) 101.

[4] F. Englert and R. Brout, Phys. Rev. Lett. 13 (1964) 321; P.W. Higgs, Phys. Lett. 12 (1964) 132; G.S. Guralnik, C.R. Hagen, and T.W. Kibble, Phys. Rev. Lett. 13 (1964) 585 .

[5] R. Gastmans, S.L. Wu, and T.T. Wu, CERN-PH-TH/2009-131, July 2009, unpublished.

[6] H. Cheng and T.T. Wu, Expanding Protons: Scattering at High Energies (MIT Press, Cambrige, 1987).

[7] A. Schäfer, O. Nachtmann, and R. Schöpf, Phys. Lett. B 249 (1990) 331.

[8] B. Müller and A.J. Schramm, Nucl. Phys. A 523 (1991) 667.

[9] A. Bialas and P.V. Landshoff, Phys. Lett. B 256 (1991) 540; J.-R. Cudell and O.F. Hernández, Nucl. Phys. B 471 (1996) 471; V.A. Khoze, A.D. Martin, and M.G. Ryskin, Phys. Lett. B 401 (1997) 330; Eur. Phys. J. C 14 (2000) 525; D. Kharzeev and E. Levin, Phys. Rev. D 63 (2001) 073004; M. Boonekamp, R. Peschanski, and C. Royon, Phys. Rev. Lett. 87 (2001) 251806; N. Timneau, R. Enberg, and G. Ingelman, Acta Phys. Polon. B 33 (2002) 3479; B. Cox, J.R. Forshaw, and B. Heinemann, Phys. Lett. B 540 (2002) 263; A. Bzdak, Phys. Lett. B 615 (2005) 249.

[10] R. Gastmans, S.L. Wu, and T.T. Wu, Phys. Lett. B 683 (2010) 354.

[11] R. Gastmans, S.L. Wu, and T.T. Wu, CERN-PH-TH/2009-132, July 2009, unpublished.

[12] C. Bourrely, J. Soffer, and T.T. Wu, Phys. Rev. D 19 (1979) 3249; Nucl. Phys. B 247 (1984) 15.

[13] C. Bourrely, J. Soffer, and T.T. Wu, Phys. Lett. B 196 (1987) 237; Z. Phys. C Particles and Fields 37 (1988) 369; Eur. Phys. J. C 28 (2003) 97.

[14] S. Mandelstam, Phys. Rev. 112 (1958) 1344. 
[15] T.T. Wu and C.N. Yang, Phys. Rev. B 137 (1965) 708; L. Van Hove, in Proceedings of the Conference on High-Energy Two-Body Reactions, Stony Brook, N.Y. (1966).

[16] T. Regge, Nuovo Cimento 14 (1959) 951; 18 (1960) 947.

[17] I.Ya. Pomeranchuk, Sov. Phys.- JETP 7 (1958) 499.

[18] ALEPH Collaboration, R. Barate et al., Phys. Lett. B 495 (2000) 1; DELPHI Collaboration, P. Abreu et al., Phys. Lett. B 499 (2001) 23; OPAL Collaboration, G. Abbiendi et al., Phys. Lett. B 499 (2001) 38; L3 Collaboration, P. Achard et al., Phys. Lett. B 517 (2001) 319.

[19] P.A. McNamara III and S.L. Wu, Rep. Prog. Phys. 65 (2002) 465.

[20] T.G. Rizzo, Phys. Rev. D 22 (1980) 178.

[21] A. Djouadi, M. Spira, and P.M. Zerwas, Phys. Lett. B 264 (1991) 440.

[22] Higher Transcendental Functions, Bateman Manuscript Project, edited by A. Erdélyi (McGraw-Hill, New York, 1953), Vol. II, p. 95, eq. (51).

[23] R. Gastmans, S.L. Wu, and T.T. Wu, Phys. Lett. B 693 (2010) 452.

[24] Particle Data Group, C. Amsler et al., Review of Particle Physics (2008).

[25] M. Koshiba, Progr. Theor. Phys. (Tokyo) 37 (1967) 1042.

[26] G.S. Abrams et al., Phys. Rev. Lett. 63 (1989) 724, 2173; L3 Collaboration, B. Adeva et al., Phys. Lett. B 231 (1989) 509; ALEPH Collaboration, D. Decamp et al., Phys. Lett. B 231 (1989) 519; OPAL Collaboration, M.Z. Akrawy et al., Phys, Lett. B 231 (1989) 530; DELPHI Collabortation, P. Aarnio et al., Phys. Lett. B 231 (1989) 539.

[27] T. Himel and J. Siegrist, in Laser Acceleration of Particles, Malibu, California, 1985, edited by C. Joshi and T. Katsouleas, AIP Conf. Proc. No. 130, (AIP, New York, 1985), p. 602; R.J. Noble, Nucl. Instrum. Methods Phys. Res. A 256 (1987) 427; M. Jacob and T.T. Wu, Nucl. Phys. B 318 (1989) 53; R. Gastmans and T.T. Wu, The Ubiquitous Photon - Helicity Method for QED and QCD, (Clarendon Press, Oxford, 1990), ch. 12.

[28] V. Barger and T. Han, Mod. Phys. Lett. A 5 (1990) 667; V.A. Ilyin, A.E. Puklov, Y. Kurihara, Y. Shinizu, and T. Kaneko, Phys. Rev. D 54 (1996) 6717; D. Djouadi, W. Kilian, M. Mühlleitner, and P.M. Zerwas, Eur. Phys. J. C 10 (1999) 27.

[29] T.T. Wu, in Proceedings of the Conference in Honor of C.N. Yang's 85th Birthday, edited by M.-L. Ge, C.H. Oh, and K.K. Phua (World Scientific, Singapore, 2008), p. 112 .

[30] M. Spira, A. Djouadi, D. Graudenz, and P.M. Zerwas, Nucl. Phys. B 453 (1995) 17. 
[31] J.C. Collins, T.C. Rogers, and A.M. Staśto, Phys. Rev. D 77 (2008) 085009.

[32] V. Sudakov, Zh. Eksp. Teor. Fiz. 30 (1956) 87; (Eng. trans.) Sov. Phys. JETP 3 (1956) 65. 